\title{
Dynamics of $\mathrm{SiO}_{2}$-glasses
}

\author{
C. Oligschleger \\ Institut für Physikalische und Theoretische Chemie, Universität Bonn, D-53115 Bonn, and Institut für Algorithmen und \\ Wissenschaftliches Rechnen, GMD - Forschungszentrum Informationstechnik, D-53754 Sankt Augustin, Germany
}

(September 13, 2021)

\begin{abstract}
Results on the dynamics of silica are presented: vibrations and relaxations. Using molecular dynamics, glass structures are generated by rapidly quenching melts below the glass transition. For the local minima of the structures the vibrational density of states is determined, the structures of the eigenmodes are analyzed, and the influence of the single components is discussed. Relaxations are studied in both amorphous $\mathrm{SiO}_{2}$ and silica melts. Our main focus is on the type of relaxation, i.e., whether the main contributions are caused by small atomic displacements, or by bond-breaking processes, e.g. creation and annihilation of dangling bonds. The eigenvector of the relaxations is found to be similar to the low-frequency one.
\end{abstract}

PACS number(s): 61.43.Fs, 66.30.Fq

\section{INTRODUCTION}

Glasses belong to the oldest manufactured materials made by man. But despite the long traditions and abilities in producing glasses still sufficient information about the atomic structures in amorphous and glassy states is lacking. Due to the absence of periodicity found in crystals both experiment and theory are challenged to work with sophisticated methods. The technological relevance of ceramics has led to increased efforts to resolve the structures and to gain insight into the so-called structureproperty-relationship which can be exploited for the design of new materials. Neutron- or x-ray-scattering provides information about the global arrangement of the atoms via the radial distribution of the pair-correlationt. Electron spin resonance and positron annihilation is used to detect dangling bonds in the structures2. From nuclear magnetic resonance studies gne can get insight into the distribution of bond angles 3 , however models are necessary to interpret the results. Due to the enormous increase in computer power, simulation has become a widely used tool to model amorphous structures. The applied techniques encompass molecular dynamics (MD) 12, Mante Carlo (MC) 1314 and molecular modelling (MM) 15 16, each of which has advantages and draw backs. Molecular modelling is succesffilly applied to covalently bonded materials, e.g. $\mathrm{SiO}_{2} 17$ and $\mathrm{GeO}_{2} 18$. Its main advantage is that, without applying refined interaction potentials, high-quality structures can be modelled which show all the experimentally known data per constructionem. The draw-back is the need of information about typical bond-lengths and bond-angles of structural units in the glass. Monte Carlo methods are applied to investigate the phase space and its properties and to study relaxations from a global point of view 19 . The Reverse Monte Carlo (RMC) technique is often used to build structures according to experimental information, e.g. neutron-scattering data20. The disadvantage of MC is that moveclasses may often be unphysical and do not give reliable insight into the microscopic dynamics of the systems. Molecular dynamics is widely used to construct models of amorphous state by rapidly quenching melts and analyzing the dynamics of the models21. Due to the fact that high-quality interaction potentials are necessary to simulate both structure and dynamics of the materials under consideration the results of MD strongly depend on these potentials. In all cases (MM, MC and MD) the typical system sizes are of the order of several thousand atoms, which correspond to a volume of several ten thousands cubic Angstroms. These small system sizes cannot be used to simulate large scale effects, e.g. grain boundaries (where a grain would be of the order of a typically available model size).

Compared to crystalline phases amorphous materials show a richer dynamics which plays an important relp with respect to low temperature thermal properties 22.23 . Apart from the long wavelength modes (sound waves) also present in crystals, in glasses strongly overdamped modes are observed at low frequencies. These strongly overdamped modes lead to a strong reduction of the thermal conductivity in glasses compared to their crystalline analogues. These modes can also be seen in the excess specific heat at low temperatures . typically below a few K.24 In the corresponding crystals one finds an increase of the specific heat with $T^{3}$ according to the Debye mode 25.26. The low temperature behaviour of glasses is well described by the tunneling model assumino a constant distribution of two-level tunneling states2728.

At somewhat higher temperatures the specific heat shows a bump in $c_{p} / T^{3}$. Using Raman29 and neutron scattering 30 one has deduced that thermally activated jumps over energy barriers and vibrations, which become anharmonic at the lowest frequencies, are responsible for such a behaviour. The influence of these effects is well described by the soft potential model (SPM) 3132 in which one assumes that the dynamics of soft regions in the structures is governed by soft potentials. The SPM is an extension of the standard tunneling model which is included in the SPM as its low temperature limit. The parameters describing the softness of both the twoand one-well potentials are distributed according to some probability distributions. Fitting these parameters to ex- 
perimental data one finds that the regions comprising the active ribating atoms are confined to about 20 to 100 atoms33. 34 .

Recently, computer simulations of soft sphere glasses (SSG) have supported the view of soft local modes 35,36 with entities comprising about 20 to 50 atoms. Similar modes have since been observed in simulations of covalently bonded glasses, e.g. $\mathrm{SiO}_{2} 37$ and $\mathrm{Se} 38$, in metallic glasses, such as Ni-Zr39, $\mathrm{Pd}-\mathrm{Si} 40$, and amorphous and quasi-crystalline Al-Zn-Mg41 and other systems, for example amorphous ice 42 . In an earlier simulation of amorphous silicon low-frequency localized vibrations have been observed at coordination defects 43 .

Further and in addition to these periodic excitations, relaxations (aperiodic rearrangements) are observed in ultrasonic and dielectric relaxation experiments 44 These processes occur already at low temperatures $(T>5 \mathrm{~K})$ and can be either tunneling processes or thermally activated jumps over energy barriers. In the SPM local relaxations are strongly correlated with local soft vibrations, assuming a smooth transition from vibrations to relaxations. Experimentally this is supported by the similar ity of the structure factors of both types of excitations 30 . From diffusion measurements in a metallic glass effective masses of ten atoms have been derived for this collective motion 45 . Strong correlations between soft vibrations and hopping have been observed in molecular dynamics simulation of the SSG46 and in amorphous Se 47 . Both reversible and irreversible relaxations were observed which consist of a collective hopping of groups of atoms. Collective hopping motions have also been observed in simulations of binary Lennard-Jones and soft-sphere mixtwres above and below the glass transition temperature 48,49 and in amorphous_Ar after introduction of vacancies 50 . Heuer and Silbey 51 systematically searched for double well potentials in a binary model glass at $T=0$. They found a distribution of two well potentials in qualitative agreement with the soft potential model.

In this paper we investigate the structure, vibrations and relaxations in amorphous silica. The following chapter is devoted to computational details. The analysis of the structures is given in section III. The vibrations and related quantities are shown in section IV. The results of a detailed analysis of the modes similar to the one given by Taraskin and Elliott52 are presented in section 凤. The study of relaxations in silica glasses and melts (section VI) is the most important part of the work presented here. To our knowledge this is the first extensive such study. As done in previous work on SSG and Se, the relaxations are analyzed with respect to jumplength, localization and element specific contributions to the atomic rearrangements. In the last section the results are discussed and conclusions are drawn.

\section{COMPUTATIONAL DETAILS}

\section{A. $\mathrm{SiO}_{2}$ and its potential}

$\mathrm{SiO}_{2}$ exists in many different crystalline allotropes (e.g. $\alpha$ - and $\beta$-quartz, high- and low-cristobalite, tridymite, keatite, coesite and stishovite) 53 . It is known to be a strong glass formert 54 .

The atomic interaction potential used in our simulation was fitted by Vashishta et al. 8 in order to reproduce structural and dynamical properties of both crystalline and glassy phases. The potential consists of two-body and three-body-terms. The two-particle interaction contains a long-range Coulomb part which we treat by an Ewald summation 55, and includes terms for steric repulsion of the particles and polarizability of the atoms.

The three-body-interaction is only relevant for Si-O$\mathrm{Si}$ and $\mathrm{O}-\mathrm{Si}-\mathrm{O}$ units, for the other possible combinations ( $\mathrm{Si}-\mathrm{O}-\mathrm{O}, \mathrm{O}-\mathrm{Si}-\mathrm{Si}, \mathrm{O}-\mathrm{O}-\mathrm{O}$, and $\mathrm{Si}-\mathrm{Si}-\mathrm{Si}$ ) it is neglected. The three-particle terms favour the tetrahedral angle at the silicon $(\mathrm{O}-\mathrm{Si}-\mathrm{O})$ and an angle $\Theta_{0} \approx 140^{\circ}$ at the corner sharing oxygen ( $\mathrm{Si}-\mathrm{O}-\mathrm{Si}$ ). For details see Ref. 8.

\section{B. Structures and Relaxations}

To produce silica glasses we quenched equilibrated melts below the glass transition temperature $T_{g}$, the number density is kept constant during all MD-runs and corresponds to a density $\rho=2.20 \mathrm{~g} / \mathrm{cm}^{3}$. An estimate of the glass-transition temperature was obtained by calculating the diffusion constant, D, of the system as a function of temperature using the relation

$$
D_{\alpha}=\lim _{t \rightarrow \infty} \frac{1}{6 t}<\left|\mathbf{R}_{\alpha}(0)-\mathbf{R}_{\alpha}(t)\right|^{2}>
$$

where $\mathbf{R}_{\alpha}(t)$ is the time-dependent position vector of a particle of type $\alpha \in\{\mathrm{Si}, \mathrm{O}\}$ and $<\ldots>$ denotes the configurational average.

In Fig. 11 the temperature dependent self diffusion constant for both silicon and oxygen shows a rapid drop at a temperature $T_{0} \approx 2500 \mathrm{~K}$. This temperature $T_{0}$ can be taken as a upper limit for the glass transition temperature $T_{g}$, since below $T_{0}$ the diffusive motion of the particles is effectively frozen out. In the high temperature limit the ratio between the oxygen and silicon diffusion constant is about 1.2 which is less than one would expect from the mass ratio $m_{\mathrm{Si}} / m_{\mathrm{O}}=1.75$ if the different species diffuse according to their respective momentum distribution. But this effect might be explained by the different "self"-interaction term in the Si-Si- and O-O-interaction. In comparison to experiment (where one measures $T_{g} \approx 1500 \mathrm{~K} 54$ ) the calculated glass transition temperature is much too high. This problem is common to all computer simulations and caused by too high quench rates, which exceed the experimental ones by many orders of magnitude 12 .

We used constant quench rates of $\dot{T}<3 \cdot 10^{13} \mathrm{~K} / \mathrm{s}$ and quenched the glasses in two steps: first we quenched the 
systems from $T=3500 \mathrm{~K}$ to $1700 \mathrm{~K}$, monitored relaxations and jumps in a temperature regime well above and below $T_{g}$, and stored the atomic positions of the detected new minima. In a next step the temperature of these glasses are quenched with the same rate to $270 \mathrm{~K}$. Again we monitored the relaxations and stored the atomic coordinates. We have generated 14 glasses with $N=1944$ atoms each. To study system size effects we have additionally constructed 10 smaller glasses with $N=576$. The time step in our simulations is about $1.2 \mathrm{fs}$. Surface effects are reduced by applying periodic boundary conditions. During equilibration we used the NVT ensemble.

The resulting structures are used to analyse relaxations both around the glass transition temperature $T_{g} \approx$ $2500 \mathrm{~K}$ and well below. To detect the relaxations in the course of the MD runs we monitored the atomic displacements,

$$
\Delta R^{2}(t)=\sum_{n}\left(\mathbf{R}_{n}(t)-\mathbf{R}_{n}^{i}\right)^{2}
$$

where $\mathbf{R}_{n}(t)$ is the position vector of atom $n$ and $\mathbf{R}_{n}^{i}$ gives its position in minimum $i$ of the potential energy surface. If the total displacement of the atoms exceeded a cut-off value, and the residence time of the atoms in the new positions also exceeded a minimal period of at least three times the period of a typical soft vibrational mode, the new positions of the particles were accepted as a new minimum configuration. The cut-offs of displacement and resident time, respectively, are chosen to avoid spurious minima. Relaxations were observed by monitoring the total displacement with respect to the initial minimum, see Eq. 2, the corresponding minima on the potential energy surface, which are identified by a final quench to $T=0 \mathrm{~K}$, were stored and analysed.

To study the relaxations in glasses at elevated temperatures, the quenched glasses were heated to temperatures between $270 \mathrm{~K}$ and $1670 \mathrm{~K}$. At each temperature the glasses were observed for up to 250000 time-steps, corresponding to about $0.3 \mathrm{~ns}$. To keep the temperature constant we averaged $T\left(t_{i}\right)$ over a period of 20 time steps $\left[T_{a v}=\frac{1}{20} \sum_{i=m}^{m+20} T\left(t_{i}\right)\right]$ and scaled the velocities after each period by $\sqrt{T_{0} / T_{a v}}$ with $T_{0}$ being the "desired" temperature and $T_{a v}=$ the averaged temperature. This has the effect that potentially relaxing atoms are not "slowed down" by reducing their velocities.

\section{Vibrations}

After a final quench of the low temperature configurations to $0 \mathrm{~K}$ by a steepest descent/conjugate gradient algorithm 1 the minima of the potential hypersurface are identified. In these minima, we calculated the dynamic matrix, diagonalized it and determined its eigenvalues, the latter correspond to the squares $\left(\omega^{2}\right)$ of the eigenfrequencies.57 The numerically exact minimization of the potential energy-prevents the occurrence of spurious unstable modes 6. The elements of the dynamic matrix are given by

$$
D_{\alpha \beta}^{m n}=\frac{1}{\sqrt{M_{n} M_{m}}} \frac{\partial^{2} U\left(\left|\mathbf{R}^{m}-\mathbf{R}^{n}\right|\right)}{\partial R_{\alpha}^{m} \partial R_{\beta}^{n}}
$$

which are the mass-weighted second derivatives of the potential energy $U$ with respect to the atomic positions. The frequency spectra are calculated from the frequencies of the $3 N-3$ vibrational modes $\sigma$ as

$$
Z(\nu)=\left\langle\frac{1}{3 N-3} \sum_{\sigma} \delta\left(\nu-\nu^{\sigma}\right)\right\rangle
$$

where $\delta$ is the discretized $\delta$-function and $\langle\ldots\rangle$ stands for the averaging over configurations. Due the limited system size the spectrum is cut-off at small frequencies. To close this gap we calculated the Debye spectrum

$$
Z_{\text {Debye }}=\frac{3}{\nu_{D}^{3}} \nu^{2}
$$

with

$$
\nu_{D}=\bar{c}\left(\frac{3 N}{4 \pi V}\right)^{1 / 3}
$$

and the average sound velocity $\bar{c}$ given in terms of the longitudinal and transverse velocities $c_{\ell}$ and $c_{t}$. These are calculated from the elastic constants of the glass by the usual relation $c_{\ell}=\sqrt{c_{11} / \rho}$ and $c_{t}=\sqrt{c_{44} / \rho}$ where we employ the elastic isotropy of the glass.

We calculated the elastic constants from the change in potential energy, $\Delta E$ under an applied strain

$$
\begin{gathered}
R_{\alpha}^{m} \rightarrow R_{\alpha}^{m}+\sum_{\beta} \epsilon_{\alpha \beta} R_{\beta}^{m} . \\
\Delta E=-\sum_{\alpha \beta} P_{\alpha \beta} \epsilon_{\alpha \beta}+\frac{V}{2} \sum_{\alpha \beta \gamma \delta} \epsilon_{\alpha \beta} C_{\alpha \beta \gamma \delta} \epsilon_{\gamma \delta}+\frac{1}{2} \sum_{\alpha \beta \gamma} P_{\alpha \beta} \epsilon_{\alpha \gamma} \epsilon_{\gamma \beta} .
\end{gathered}
$$

Here the first term accounts for the work done against the forces for an ensemble which is not in equilibrium against volume changes where $P_{\alpha \beta}$ is the virial of the forces. The third term is a correction for the volume change under a finite shear in such a lattice and the $C_{\alpha \beta \gamma \delta}$ are the elastic constants $\left(c_{11}=C_{1111}, c_{44}=C_{2323}\right)$.

We found for the glass the sound velocities $c_{\ell}=$ $6100 \mathrm{~m} / \mathrm{s}$ and $c_{t}=4600 \mathrm{~m} / \mathrm{s}$ which are about $10 \%$ and $20 \%$ higher than the experimental values. Taking the finite size of the ensemble into account this implies that the lowest frequency sound wave which could be observed for the largest sample $(\mathrm{N}=1944$ atoms $)$ is about $\nu \approx 1.5 \mathrm{THz}$. 


\section{STRUCTURE OF SILICA-GLASSES}

In Table 1 the distribution of the coordination numbers $\mathrm{CN}$ for the single components at different temperatures is given. If the atomic distances $r_{i j}<2.3 \AA$ (this distance corresponds to the minimum in the pair-correlation function, see below) then atoms $i$ and $j$ are neighbours. One can clearly observe a reduction of the fraction of "coordiantion defects", i.e. atoms with another coordination number than the most common one, with decreasing temperature. During cooling the local topology of the atoms improves due to relaxation of the structures. In the liquid phase at $T=3500 \mathrm{~K}$ nearly $10 \%$ of the particles are under- or over-coordinated. Whereas equal fractions of oxygen atoms are under- and over-coordinated, nearly no over-coordinated silicon is found. However, in the glassy state at $T=870 \mathrm{~K}$ the fractions of under-coordinated $\mathrm{Si}$ and $\mathrm{O}$ are reduced to less than $5 \%$ and less than $3 \%$, respectively. Over-coordinated oxygen atoms have nearly disappeared, and nearly no over-coordinated silicon is found. In the glasses quenched from $T=270 \mathrm{~K}$ to $T=0 \mathrm{~K}$ the total fraction of defects is even less than $3 \%$. From experiments it is known that in silica coordination defects exist in which the silicon is only surrounded by three oxygen (oxygen vacancy) 59 the number of these defects is enhanced by irradiation. Our numbers of coordination defects are comparable to other models and simulations. In molecular dynamics simulation of silica glasses the numbers of defects can vary between less than $2 \%$, 12 and $6 \%$ to $8 \%$ as pointed out in Ref. 6 and references therein, the effective coordination number strongly depend gn the applied interaction potentials 6 and quenchrates 12 . Using molecular modelling techniques vitreous structures withqut any defects can be constructed per definitionem 17 18. Typically these structures are cluster models, i.e. without periodic boundary conditions.

Quantities well-suited to gain insight into structural correlations and comparing simulations and experiments are the total and partial pair-correlation functions. We take the usual definition of the partial pair-distribution functions $g_{\alpha \beta}(r)$

$$
<n_{\alpha \beta}(r)>\Delta r=4 \pi r^{2} \Delta r \rho_{N} c_{\beta} g_{\alpha \beta}(r)
$$

where $n_{\alpha \beta}(r) \Delta r$ is the number of particles of species $\beta$ in a shell of thickness $\Delta r$ and radius $r$ around a particle of species $\alpha$ and $\langle\ldots\rangle$ denotes the configurational average. $\rho_{N}=N / V$ is the number density and $c_{\beta}=N_{\beta} / N$ the concentration of species $\beta$. The partial pair-correlation functions obtained at $T=270 \mathrm{~K}$ shown in Fig. 2(bd) are used to assign the peaks in the total $g(r)$, Fig. 2 (a), to the partial contributions. From the integral over the first maximum of the pair-correlation functions one can deduce average coordination numbers: each silicon has 3.96 oxygen neighbors. The coordination numbers of the O-O-correlation reaches a number 6 for the range from $0.25 \mathrm{~nm}$ to $0.3 \mathrm{~nm}$. For $\mathrm{Si}-\mathrm{Si}$ we observe a value of 4 in the range from $0.28 \mathrm{~nm}$ to $0.35 \mathrm{~nm}$. From these partial pair-correlations one can deduce that the peak at $0.42 \mathrm{~nm}$ with a shoulder at about $0.35 \mathrm{~nm}$ is a superposition of the second peak in the Si-O-contribution and the shoulder of the second O-O-peak. The fifth peak in the pair-distribution function at $0.5 \mathrm{~nm}$ in Fig. 2 appears to be a superposition of the second peaks of the O-O- and Si-Si-pair correlations.

In Fig. 3 the pair-distribution function $d(r)=$ $4 \pi r \rho_{N}(g(r)-1)$ averaged over an ensemble of 14 glasses with $N=1944$ atoms is shown.Together with the result of an electron scattering experiment 60 . Up to For $r \approx 0.6 \mathrm{~nm}$, i.e. twice the average Si-Si-distance, both curves agree very well. (The wiggles below the first peak in the experimental curve are an artefact.)

The bond-angle distributions are plotted in Fig. 4. The O-Si-O distribution has its peak-position at $109^{\circ}$ and a full width at half maximum (FWHM) of $10^{\circ}$. Such a narrow distribution of the tetrahedron-angle suggests slightly distorted tetrahedra as basic structural unit. The Si-O-Si distribution shows a peak at $145^{\circ}$ and a FWHM of $25^{\circ}$. This broader angle distribution refelcts the disorder in the silica glasses. In the O-O-Si angle distribution the peak at $35^{\circ}$ stems from atoms belonging to the same tetrahedron. It is directly connected to the sharp peak in the O-Si-O angle distribution. The broad distribution between $80^{\circ}$ and $180^{\circ}$ stems from configurations where the two O-atoms have different Si's as nearest neighbours. In the $\mathrm{Si}-\mathrm{Si}-\mathrm{O}$ angle distribution the peak around $20^{\circ}$ stems from two silicon atoms bonded to the same oxygen. This peak is directly connected to the $\mathrm{Si}-\mathrm{O}-\mathrm{Si}$ distribution. If an oxygen is not a bridging atom between two silicon atoms, but instead neighbouring the adatom, we find an angle between $60^{\circ}$ and $160^{\circ}$. The strong peak at $60^{\circ}$ in the O-O-O angle distribution is from triplets of O's bonded to the same Si-atom. For a situation in which the oxygen atoms are bonded to different Si the O-O-O angles are always larger. For the Si-Si-Si angle we find a broad distribution between $80^{\circ}$ and $180^{\circ}$ with a maximum at about $105^{\circ}$. This might give a hint to patterns in which the silicons are surrounded by strongly distorted "Si-tetrahedra". The distance between the next-nearest silicons in the triple is about $0.5 \mathrm{~nm}$. In triples where all $\mathrm{Si}$ are at their nearest neighbor distance $(\approx 0.3 \mathrm{~nm})$ we find Si-Si-Si angles of about $60^{\circ}$ which explains the small vicinal maximum in the distribution. This points to the existence of 6-membered Si-O-rings in silica. In all, the angle distribution clearly reveals corner-sharing tetrahedra of $\mathrm{SiO}_{4}$.

\section{SPECTRA AND LOCALIZATION OF VIBRATIONAL MODES}

Using Eq. 1 we show the vibrational density of states as $Z(\nu)$ in Fig. 5 averaged over all configurations together with the Debye spectrum, Eq. 5. One sees a clear enhancement of the glassy spectrum at low frequencies 
which reaches well beyond frequencies where system size could be of importance. Reflecting some shortcomings of the interaction potential the experimentally observed double peak at high frequencies 61 cannot be reproduced. The Bose-peak, i.e. the maximum of $Z(\nu) / \nu^{2}$, lies at $\approx$ 1.6 $\mathrm{THz}$ higher than experiment where one observes it at about 1 THz62. From the density of states we can calculate the vibrational specific heat at constant volume $c_{v}$. In the harmonic approximation one has per atom

$$
c_{v}=3 k_{B} \int d \omega\left[\left(\frac{\hbar \omega}{2 k_{B}}\right)^{2} / \sinh ^{2}\left(\hbar \omega / 2 k_{B} T\right)\right] Z(\omega) .
$$

The vibrational specific heat of a perfect crystal at low temperatures is $\propto T^{3}$. It is, therefore, usual practice to plot $c_{v} / T^{3}$. In such a plot a Debye spectrum gives a constant whereas in glasses an increase above this constant is found.

Fig. 6 shows this behavior for the silica glasses. The solid line shows the values gained from the spectrum of Fig. 1 which is corrected for the finite size of the simulated glass by adding a Debye contribution up to a frequency smaller than the lowest possible phonon frequency. This correction amounts to a fraction of $7.7 \times 10^{-3}$ of all modes. The resulting values for the specific heat are shown by the full line. The diamonds corresponds to experimental values 24 . The too high values of the sound velocities in our model cause a too high value of $\nu_{D}$ which leads to a Debye contribution to $c_{V}$ which is too small by a factor of $\approx 1.6$.

The phonon eigenvectors describe the structure of the vibration and can, e.g. be used to determine the degree of the localization of the vibration. There are two commonly used measures of localization, the effective mass and the participation ratio 15.35 . The effective mass is given in terms of the eigenvector as

$$
m_{\mathrm{eff}}(\sigma)=m /\left|\mathbf{e}^{\mathbf{1}}(\sigma)\right|^{2}
$$

Here we have assumed that the $3 N$-dimensional unit vector of mode $\sigma$ is normalized and $\mathbf{e}^{n}(\sigma)$ stands for the vector formed from the three components on atom $n$. Atom number 1 is chosen as the atom with the largest displacement. $m_{\mathrm{eff}} / m$ is a measure for the number of atoms which effectively carry the kinetic energy of the vibrational mode. This definition is limited to small system sizes when the long range tails of the modes are not too important. In the following we will mainly use the participation ratio:

$$
p(\sigma)=\left(N \sum_{n=1}^{N}\left|\mathbf{e}^{n}(\sigma)\right|^{4}\right)^{-1}
$$

For a translation one has $p=1$ and for a vibration of a single atom with all others at rest $p=1 / N$. This scaling with $1 / N$ should hold for all localized modes. Fig.
7 shows the participation ratios for the two system sizes with $N=576$ and $N=1944$ atoms. The high-frequency modes are highly localized and their participation ratios show the expected scaling with system size. At the lowfrequency end we observe (quasi-)localized modes. The localization corresponds to clusters comprising about 10 to 50 atoms. The strongest localized modes have participation ratios of 0.012 corresponding to an entity comprising about 8 atoms These findings are in agreement with ones by Jin et al 37 and Taraskin and Elliott52. However, their participation ratios do not show the expected $1 / N$ scaling. This will be due to interaction effects between the modes in the larger structures which results in an increase of the participation ratio. In the larger systems there are more of similar frequency, and in particular more low frequency extended phonons which will mix with the quasi-localized modes and raise their participation ratio. Additionally this causes an increased interaction between quasi localized modes of similar energy, since due to de-localization these modes lose their "splendid isolation" and "feel" also other localized vibrations, too. Due to this interaction of quasi localized modes their participation ratio is increased. For a finite concentration $c$ of interacting modes the scaling factor $1 / N$ in Eq. 12 will be replaced by $c$. Such effects have also been found in a model glass 63 where the modes are deconvoluted such as to reconstruct the "naked" contributions: phonons and localized vibrations.

\section{ANALYSIS OF VIBRATIONAL MODES}

To gain more insight into the dynamics of the vibrational modes we analysed the motion of the single components and their contribution to the eigenvectors. The averaged contribution of the oxygen to the mass-weighted eigenmodes in comparison with the silicon is depicted in Fig. 8. This contribution is given as the mean square displacement of each component $\alpha \in\{\mathrm{Si}, \mathrm{O}\}$ to the eigenvectors

$$
Z^{\alpha}(\nu)=\left\langle\frac{1}{3 N-3} \sum_{\sigma} \delta\left(\nu-\nu^{\sigma}\right) \sum_{i=1}^{N_{\alpha}}\left(e_{i}^{\sigma}\right)^{2}\right\rangle,
$$

with $N_{\alpha}$ the number of the $\mathrm{Si}$ - and O-atoms, respectively. Just below the high-frequency peak the silicon atoms contribute stronger to the eigenvectors than the oxygen atoms, whereas the eigenmodes of the low-frequency part of the spectrum and the high-frequency modes are clearly dominated by the oxygen motion. To learn about typical motions we calculated the angle between the displacement of atom $i$ in the modes $\left(\mathbf{e}_{i}\right)$, and the bonds of this atom to its nearest neighbors $j$, i.e. $\mathbf{r}_{\text {bonds }}=\mathbf{r}_{i j}$

$$
\alpha_{e_{i} \text { bonds }}=\arccos \left(\frac{\mathbf{e}_{i} \mathbf{r}_{\text {bonds }}}{\left|\mathbf{e}_{i}\right|\left|\mathbf{r}_{\text {bonds }}\right|}\right)
$$

In Fig. 9 we show the weighted distribution of the angles for the low-frequency modes of each glass with 
respect to the components $\mathrm{Si}$ and $\mathrm{O}$. We weighted with $e_{i}^{2}$ in order to suppress marginal contributions of only slightly moving atoms. The contributions of both elements are peaked at $90^{\circ}$ which means the motion of both $\mathrm{Si}$ and $\mathrm{O}$ is mainly perpendicular to the bonds. In the case of oxygen this peak is very sharp, whereas the silicon atoms have stronger contributions to either smaller or larger angles, this indicates that the silicon atoms have contributions parallel to their bonds. Following the work by Taraskin and Elliott 52 we calculated the projections of the vibrations of the structural subunits, such as the $\mathrm{SiO}_{4}$-tetrahedra and the $\mathrm{SiOSi}$-units, onto the symmetry vibrational coordinates, i.e. internal vibrations, such as symmetric and asymmetric stretching, bending modes, and rigid body motions, namely rotations and translations. The translations of the sub-units are neglible $(<1 \%)$ in comparison to rotations and internal vibrations. To calculate the internal motions the center of these subunits are fixed, i.e. the vibrations of the surrounding atoms are shifted relative to the central atoms. The remaining motions can be either rigid rotations or internal vibrations, such as stretching or bending modes. In Fig. 10 we show the contributions of the projections onto the SiOSi subunits to the total DOS. The modes in the low-frequency range have a strong rotational character, whereas in the high-frequency peak an asymmetric stretching mode describes well the typical vibrations. In the mid part of the spectrum symmetric bending and with less extension also symmetric stretching contribute to the modes. Projecting the modes onto the symmetry vibrations of the $\mathrm{SiO}_{4}$ structural subunits (see Fig. 11) we find the high-frequency peak to be described well by both asymmetric (strong contribution) and symmetric (weak contribution) stretching modes. Sipce in the experimental DOS one observes a double peak 61 , we can speculate that our interaction potential gives a too low concentration of symmetric stretching modes and a too high concentration of asymmetric modes. The center of the spectrum has contributions from bending modes with $\mathrm{F}_{2}$ and E symmetry. The low-frequency side of the spectrum is again dominated by rotations. This is in agreement with the observation that the atoms move perpendicularly to their bonds.

\section{RELAXATIONS ABOVE AND BELOW THE GLASS TRANSITION TEMPERATURE}

At the start of the simulations the temperature of the configurations is about $3500 \mathrm{~K}$. Cooling with a quench rate of about $\dot{T}<3 \cdot 10^{13} \frac{\mathrm{K}}{\mathrm{s}}$ the structures cooled to $T=1700 \mathrm{~K}$ after 50000 time steps $\approx 60$ ps. During cooling we observe initially a rapid succession of strong relaxations. After about $20 \mathrm{ps}$ and at a temperature of circa $2800 \mathrm{~K}$ ( slightly above $\mathrm{T}_{g}$ ), the activity of the structures slows down, i.e. jumps become less frequent and the systems become temporarily trapped in metastable regions of configuration space.
During cooling several new local minima of the potential energy surface are visited by the "MD-walker" via jumps over energy barriers. To determine the localization of the hopping processes and to test the SPM assumptions, we calculated similar to Eq. 11 the effective mass of the relaxation:

$$
M_{\mathrm{eff}}=m_{\max } \cdot \frac{(\Delta R)^{2}}{\left|\Delta \mathbf{R}_{\max }^{2}\right|}
$$

where $(\Delta R)^{2}=\sum_{n}\left(\mathbf{R}_{n}^{i}-\mathbf{R}_{n}^{f}\right)^{2}$ is the square of the total distance $\Delta R$ between two successive minimum configurations (called "initial" and "final" positions of the jump). $\mathbf{R}_{n}^{i, f}$ denotes the respective initial and final positions of atom $n,\left|\Delta \mathbf{R}_{\max }^{2}\right|$ is the maximal distance a single atom jumps in this relaxation, and $m_{\max }$ is the mass of the farthest jumping atom. Similarly to Eq. 12 we calculated the participation ratio $p_{\Delta R}$ to determine the localization of the hopping processes,

$$
p_{\Delta R}=\frac{\Delta R^{4}}{N \sum_{n}\left(\mathbf{R}_{n}^{i}-\mathbf{R}_{n}^{f}\right)^{4}}
$$

where $\mathbf{R}_{n}^{i}$ and $\mathbf{R}_{n}^{f}$ denote the initial and final position of atom $n$ in the relaxation and $\Delta R$ is the total jumplength. The participation ratio has the value $n / N$ if $n$ atoms are involved in the hopping process, and $p_{\Delta R}=1$ if all atoms contribute equally to the relaxation. We find that the participation ratio of jumps grows roughly linearly with the jump distance, Fig. 13. Comparing the results for the two temperature intervals one clearly observes an increase of both $\Delta R$ and $p_{\Delta R}$ with temperature. The gap between the two temperature regimes is an artifact of the cut-off procedure used for the higher temperatures.

To study relaxations in a "metastable equilibrium" we heated the quenched glasses to $T=270,870$ and $1670 \mathrm{~K}$. For the jumps of the glasses which are heated from $T=$ $0 \mathrm{~K}$ to the desired temperature we monitored again the minima visited in course of the MD runs and determined the corresponding jumps and their participation ratios.

As example we plotted the time evolution of the energy and the displacement in Fig. 14 for the glasses at $T=$ $870 \mathrm{~K}$. In the low-temperature regime we can follow an overall relaxation of the structures towards more stable configurations. This trend becomes even stronger in the higher temperature regime. The ensemble-averaged participation ratios for the equilibrated temperatures (equilibrated during heating) show a slight temperature dependence, $p_{\Delta R}=0.020 \pm 0.008,0.057 \pm 0.035,0.061 \pm 0.036$ for $T=270,870$, and $1670 \mathrm{~K}$, respectively. Quite in contrast though, the jump-lengths increase linearly with temperature, and we find $\Delta R=1.81 \pm 0.75,4.72 \pm 1.38$, and $8.27 \pm 2.49 \AA$ for $T=270,870$, and $1670 \mathrm{~K}$, respectively. The typical "resident time" per minimum is about 15 ps. In studies of amorphous Se the resident times per minimum is found to be about $30 \mathrm{ps}$ at $0.05 T_{g} .64$

To identify active regions we calculated a correlation between the observed relaxations 


$$
c_{R R^{\prime}}=\frac{\sum_{n} \Delta R_{n} \Delta R_{n}^{\prime}}{\Delta R \Delta R^{\prime}} .
$$

Since we calculated the product of the absolute values $\Delta R$ and not the scalar product between the two jumpvectors $\boldsymbol{\Delta} \mathbf{R}$ and $\boldsymbol{\Delta} \mathbf{R}^{\prime}$, we also observed active regions where almost the same atoms contributing to a relaxation $\boldsymbol{\Delta} \mathbf{R}^{\prime}$ jump perpendicularly to the previous relaxation $\Delta R$. In that case $c_{R R^{\prime}}$ is close to 1 . If the jumps are uncorrelated $c_{R R^{\prime}}$ becomes $n / N$, with $n$ the typical number of atoms contributing to the relaxations, and $N$ the total number of atoms in the configuration.

Calculation of the correlation between successive jumps (see Eq. 17) at the lowest considered temperature $(T=270 \mathrm{~K})$ yields values of $0.585 \pm 0.314$. Astonishingly, for the glasses at $T=870$ and $1670 \mathrm{~K}$ very similar values ( $0.654 \pm 0.089$ and $0.666 \pm 0.082$, respectively) are observed. The broader distribution of values in the lowtemperature simulation may be explained by the observation that at the lowest temperature successive relaxations are either reversible jumps (high correlations) or jumps where different parts of the active regions of the systems contribute to the relaxations at different times (low correlations). At higher $T$ these local regions aggregate to a larger complex where the single entities are not resolved in the jump-process. This can explain the relatively high correlations between the jumps at higher $T$ where several "active" regions in the glasses jump at the same time. However, at the higher temperatures successive jumps are not reversible, but the same (local) regions are activated throughout the observation time.

To see whether there exists a typical "relaxationmode" we calculated the projection of the relaxations observed at $T=870 \mathrm{~K}$ and $1670 \mathrm{~K}$ onto the bonds of the atoms, Fig. 15.

$$
\alpha_{r_{i} \text { bonds }}=\arccos \left(\frac{\Delta \mathbf{R}_{i} \mathbf{r}_{\text {bonds }}}{\left|\Delta \mathbf{R}_{i}\right|\left|\mathbf{r}_{\text {bonds }}\right|}\right)
$$

where $\boldsymbol{\Delta} \mathbf{R}_{i}$ is the relaxation vector of atom $i$. The distribution is Gaussian in the case of the oxygen, and centered around $90^{\circ}$ indicating that the $\mathrm{O}$-atoms relax mainly perpendicularly to the bonds. For the silicon atoms we observe a peak at low angles which reflects the fact that the Si-atoms relax mainly in direction of one bond. Since a silicon is surrounded tetrahedrally by the O-atoms, the projection onto the remaining bonds will give the tetrahedron angle at about $109^{\circ}$, which is in satisfying agreement with the peak at about $105^{\circ}$. Note that the ratio between the peaks at $5^{\circ}$ and $105^{\circ}$ is about 1:3 which is the ratio one would expect in a tetrahedron if the center atom moves parallel to one of its bonds. In analogy to the mode-analysis we calculated the projections of the relaxations onto the symmetry modes of SiOSi- and $\mathrm{SiO}_{4}$-subunits. Since in this study it has been important to consider the "real" contributions of the atoms and not only the "relative" displacements as in the case of the eigenmodes we do not subtract the displacement vector of the central atom. In the case of the $\mathrm{SiO}_{4}$-subunits and relaxations at $T=870 \mathrm{~K}$ and $1670 \mathrm{~K}$ we do find nearly no translations of the subunit worth mentioning and negligible contributions for symmetric stretchings of the oxygen atoms; 0.21 of the relaxations of the oxygen atoms stem from asymmetric stretching, 0.40 of the jumps are due to bendings in $\mathrm{F}_{2^{-}}$ symmetry, 0.07 are bending with E-symmetry and 0.32 of the oxygen motions come from rotations. Considering the SiOSi-subunits we do not record a considerable amount of translations of the SiOSi-pattern; and only 0.037 of the Si-relaxations are due to symmetric stretching. The contribution of asymmetric stretching is about 0.26 , symmetric bendings contribute at about 0.23 to the jumps, and 0.47 come from rotations. The strong contributions from bending and rotations for $\mathrm{Si}$ and $\mathrm{O}$ are in agreement with the picture that the typical relaxation is perpendicular to the bonds. For the relaxations of silicons (in the SiOSi-subunits) we find that about $30 \%$ of the relaxations can be ascribed to stretching (3.7\% symmetric and $26 \%$ asymmetric), for the oxygen relaxations in the $\mathrm{SiO}_{4}$-units only $21 \%$ of the relaxations can be described by asymmetric stretching.

The investigation of relaxations with respect to bondchanges (creation and annihilation), where a bond is cut off at $2.3 \AA$, at constant temperatures shows that at $T=270 \mathrm{~K}$ the main contribution to a relaxation is due to small changes of the positions of the atoms, bondbreaking occurs only in a few cases. At $T=870 \mathrm{~K}$ about $0.1 \%$ of the bond situation is altered by relaxations from one minimum to another. At the highest temperature the number of bonds change up to $0.3 \%$ per jump.

During the cooling runs we stored configurations for some of the glasses at $T=870 \mathrm{~K}$ and $T=270 \mathrm{~K}$. After an equilibration time of 60 ps we started relaxation runs for these "young glasses" at $T=870 \mathrm{~K}$ and $T=270 \mathrm{~K}$, respectively. Again relaxations were monitored for about $0.3 \mathrm{~ns}$.

The participation ratios averaged over the ensembles for the equilibrated temperatures $T=270 \mathrm{~K}$ (4 glasses) and $T=870 \mathrm{~K}$ (2 glasses) are $p_{\Delta R}=0.020 \pm 0.011$ and $p_{\Delta R}=0.047 \pm 0.030$, respectively. The jump-lengths increase with temperature, we find $\Delta R=2.05 \pm 0.77 \AA$, $5.86 \pm 2.15 \AA$ again for $T=270 \mathrm{~K}$ and $870 \mathrm{~K}$, respectively. Now the typical "resident time" per minimum is about 10 ps. Note that the jump-lengths of the "young" glasses are larger and the resident time is smaller than in the corresponding "older" glasses. These results although based on weaker statistics may point to the fact that the "young glasses" are more active in terms of longer jumpdistances and shorter relaxation-times than those glasses aged for a longer time.

\section{DISCUSSION AND CONCLUSION}

We have performed molecular dynamics calculations on silica glasses and melts. The amorphous structures 
were generated by rapid quenches of melts. The analysis of the glasses show a satisfying agreement with experimental results for the pair-correlation functions. The angle-distributions reveal the existence of a network of corner-sharing tetrahedra. During cooling we observed an appreciable optimization of the local topology of the structures. At $T=3500 \mathrm{~K}$ the number of dangling bonds of $\mathrm{Si}$ and $\mathrm{O}$ is about $9.4 \%$ and $4.5 \%$, respectively. Caused by structural relaxations the number of these defects decreases to $3.9 \%$ and $2.2 \%$, respectively, at $T=0 \mathrm{~K}$. This improvement can also be seen in the shift of the peak in the Si-Si pair-correlation function from less than $0.3 \mathrm{~nm}$ in the liquid, in which the Si-Si-distance appears as a shoulder of the O-O-peak, to $0.31 \mathrm{~nm}$ (at $T=270 \mathrm{~K}$ ). The Si-Si-distance becomes visible as a separate peak in the total pair-correlation function.

One main focus of our study concerns the dynamics of the structures with respect to both periodic motions (vibrations) and aperiodic ones (relaxations). Calculation of the element specific contributions shows the silicon motion to be dominant just below the high frequency modes (about $28 \mathrm{THz}$ ). As to the rest of the spectra, the oxygen vibrations become the main contribution to the mode. The participation ratios show that all modes in the highfrequency regime are localized, comprising entities with less than 15 atoms. At the lowest frequencies the modes - about $0.2 \%$ of the total spectrum - are typically quasilocalized vibrations with participation ratios of less than 0.22 for $N=1944$ atoms. The effective numbers of atoms contributing to the quasi-localized modes range from 10 to 50 particles. The atoms strongly participating in those low-frequency modes move preferentially perpendicularly to their bonds. Investigating the subunits of the structures such as $\mathrm{SiO}_{4}$-tetrahedra and "SiOSi-fragments" we find that the motion of the low-frequency modes can be described by rotations (most important feature) with an addition of bending type modes. This picture is in agreement with the one of rotations of coupled tetrahedra 30. The high-frequency vibrations can be described well by stretching modes.

We studied relaxations of $\mathrm{SiO}_{2}$ structures in the melt (during cooling) and in the glass at several temperatures both during cooling and "equilibration". During cooling from 3500 to $1700 \mathrm{~K}$ we observed rearrangements of the structures with participation ratios of less than 0.3 (for $N=1944$ atoms) connected to effective masses ranging from 20 to 150 which are somewhere between clear local relaxations and global changes. This observation indicates that several regions of the structure are active at the same time. Lowering the temperature (from $1700 \mathrm{~K}$ to $300 \mathrm{~K}$ ) the picture of locally changing structures becomes clearer and the participation ratio of the jumps is less than 0.1. Analysis of the relaxations observed during the "equilibration" runs shows that the jumps at low temperature $(T=270 \mathrm{~K})$ typically lead to small changes of the atomic positions with nearly no bondbreaking processes. At higher $T$ we observe bond-changes of the atoms (both bond-breaking and bond-creations):
At $T=870 \mathrm{~K}$ the numbers of bonds changed by about 1 bond out of 1000 , and at $T=1670 \mathrm{~K}$ of up to 3 bonds out of 1000 changes per relaxation. These relaxations are local processes comprising typically 10-50 atoms. For the lowest temperature we find about 10 atoms contributing strongly to the relaxations whereas at higher $T$ (870 and $1670 \mathrm{~K}$ ) about 20-50 atoms are contributing. However, the jump-length increases clearly linearly with temperature. In the course of the MD simulations the structures relax to energetically lower lying minima. During aging of the structures this trend seems to slow down. Further corroboration of this lies in the observation of longer residence times of the configurations in each minimum and smaller jump-lengths towards the end of our simulations.

For all temperatures below the glass-transition we observed correlated relaxations, i.e. several active regions coupled via a few atoms contributing to the jumps. At the lowest temperature several active regions can be distinguished whereas these regions are "coupled" at higher $T$, i.e. the atoms comprising these regions are active at the same time 46,47 .

Analyzing motion patterns of the relaxations has shown that the oxygen atoms contribute dominantly to the relaxations by typically small displacements mainly perpendicular to the bonds. Nearly half of the oxygen motion is due to bond bendings and $30 \%$ to rotations (0.32). The silicon atoms exhibit also jumps more closely in the direction of one of its oxygen neighbours. This shows a more stretching-like motion of the Si-relaxation. However, the total contribution of this motion pattern amounts about only 0.3. The rest of the silicon motion can be attributed to rotations $(0.47)$ and symmetric bendings (0.23). Comparing the motion patterns of the relaxations to the eigenmodes (vibrations) the obvious similarity between the motion pattern found in both low-frequency vibrations and relaxations (main contributions are from rotations and bending) indicates a strong connection between low-frequency modes and local relaxations and supports the assumption of the soft potential model. Such a correlation between local relaxations andeigenmodes has also been found in amorphous selenium 47 However, the relaxations have stronger contributions from bending- and stretching-motions than the low-frequency modes which lead us to the conclusion that also higher-frequency modes are necessary to describe relaxations and are acting as impetus for localized jumpprocesses.

\section{ACKNOWLEDGMENTS}

The author is grateful for many stimulating discussions with U. Buchenau and H.R. Schober. Thanks are due to M. Gastreich, A. Hannemann, H. Putz and J.C. Schön for critical reading of the manuscript. Funding by the Deutsche Forschungsgemeinschaft through the SFB408 is gratefully acknowledged. 
${ }^{1}$ A.C.Wright in Experimental Technique of Glass Science, eds. C.J. Simmons and O.H. El-Bayoumi, The American Ceramic Society, Westerville, Ohio.

${ }^{2}$ A. Ogata and S.J. Tao, J. Appl. Phys. 41, 4261 (1970).

${ }^{3}$ J. Neuefeind and K.D. Liss, Ber. Bunsenges. Phys. Chem 100, 1341 (1996).

${ }^{4}$ L.V. Woodcock, C.A. Angell and P. Cheeseman, J. Chem. Phys. 65, 1565 (1976).

${ }^{5}$ F.H. Stillinger and T.A. Weber, Phys. Rev. B 31, 5262 (1985).

${ }^{6}$ B.P. Feuston and S.H. Garofalini, J. Chem. Phys. 89, 5818 (1988) and references therein.

${ }^{7}$ R. Car and M.Parrinello Phys. Rev. Lett. 60, 204 (1988).

${ }^{8}$ P. Vashishta, R.K. Kalia, J.P. Rino and I. Ebbsjö, Phys. Rev. B 41, 12197 (1990).

${ }^{9}$ S. Tsuneyuki, H. Aoki, M. Tsukada, Y. Matsui, Phys. Rev. Lett. 64, 776 (1990).

${ }^{10}$ J.S. Tse and D.D. Klug, Phys. Rev. Lett. 70, 174 (1993).

${ }^{11}$ B. Vessal, M. Amini and C.R.A. Catlow, J. Non-Cryst. Solids 159, 184 (1993).

${ }^{12}$ K. Vollmayr, W. Kob, and K. Binder, Phys. Rev. B 54, 15808 (1996), K. Vollmayr, PhD Thesis, University of Mainz (1995).

${ }^{13}$ I.N. Grossmann, Soviet Journal of Glass Physics and Chemistry 11, 384 (1985).

${ }^{14}$ V.K. Shiff, J. Non-Cryst. Solids 123, 36 (1990).

${ }^{15}$ R.J. Bell and P. Dean, Phil. Mag. 25, 1381 (1972).

${ }^{16}$ L.F. Gladden and S.R. Elliott, Phys. Rev. Lett. 59, 908 (1987).

${ }^{17}$ L. F. Gladden, J. Non-Cryst. Solids 119, 318 (1990).

18 S. Wefing, Modeling of Continous Random Networks: A Case Study for Vitreous $\mathrm{GeO}_{2}$, I., Model Generation, submitted to J. Non-Cryst. Sol., and S. Wefing, Modeling of Continous Random Networks: A Case Study for Vitreous $\mathrm{GeO}_{2}$, II., Model Refinement, submitted to J. Non-Cryst. Sol.

${ }^{19}$ R.S. Berry and R. Breitengraser-Kunz, Phys. Rev. Lett. 74, 3951 (1995).

${ }^{20}$ D.A. Keen and R.L. McGreevy, Nature 433, 423 (1990).

${ }^{21}$ M.P. Allen, and D.J. Tildesley, in: Computer Simulation of Liquids, Oxford: Clarendon Press (1987).

${ }^{22}$ W.A. Phillips, ed., Amorphous Solids: Low Temperature Properties (Springer-Verlag, Berlin 1981).

${ }^{23}$ H.-J. Günterodt and H. Beck, eds., Glassy Metals I, (Springer-Verlag, Berlin 1981).

${ }^{24}$ R.C. Zeller and R.O. Pohl, Phys. Rev. B 4, 2029 (1971).

${ }^{25}$ N.W. Ashcroft, and N.D. Mermin, Solid State Physics, (Saunders College, HRW International Editions 1987).

${ }^{26}$ H.R. Schober and W.Petry in Material Science and Technology, Vol. 1, eds. R.W. Cahn, P. Haasen and E.J. Kramer, (VCH, Weinheim 1991)

27 P.W. Anderson, B.I. Halperin and C.M. Varma, Phil. Mag. 25, 1 (1972).

${ }^{28}$ W.A. Phillips, J. Low Temp. Phys. 7, 351 (1972).

${ }^{29}$ G. Winterling, Phys. Rev. B 12, 2432 (1975).
${ }^{30}$ U. Buchenau, H.M. Zhou, N. Nücker, K.S. Gilroy and W.A. Phillips, Phys. Rev. Lett. 60, 1318 (1988).

${ }^{31}$ V.G. Karpov, M.I. Klinger and F.N. Ignatiev, Sov.Phys. JETP 57, 439 (1983).

32 M.A. Il'in, V.G. Karpov and D.A. Parshin, Sov. Phys. JETP 65, 165 (1987).

${ }^{33}$ U. Buchenau, Yu.M. Galperin, V.L. Gurevich and H.R. Schober, Phys. Rev. B 43, 5039 (1991).

${ }^{34}$ U. Buchenau, Yu.M. Galperin, V.L. Gurevich, D.A. Parshin, M.A. Ramos and H.R. Schober, Phys. Rev. B 46, 278 (1992).

35 B.B. Laird and H.R. Schober, Phys. Rev. Lett. 66, 636 (1991).

${ }^{36}$ H.R. Schober and B.B. Laird, Phys. Rev. B 44, 6746 (1991).

37 W. Jin, P. Vashishta, R.K. Kalia and J.P. Rino, Phys. Rev. B 48, 9359 (1993).

${ }^{38}$ C. Oligschleger and H.R. Schober, Physica A 201, 391 (1993).

39 J. Hafner and M. Krajčí, J. Phys.: Condens. Matter 6, 4631 (1994).

${ }^{40}$ P. Ballone and S. Rubini, Phys. Rev. B 51, 14962 (1995).

${ }^{41}$ J. Hafner and M. Krajčí, J. Phys.: Condens. Matter 5, 2489 (1993).

${ }^{42}$ M. Cho, G.R. Fleming, S. Saito, I. Ohmine, and R.M. Stratt, J. Chem. Phys 100, 6672 (1994).

${ }^{43}$ R. Biswas, A.M. Bouchard, W.A. Kamitakahara, G.S. Grest and C.M. Soukoulis, Phys. Rev. Lett. 60, 2280 (1988).

${ }^{44}$ S. Hunklinger and M. v. Schickfus in Ref.22, p.81.

${ }^{45}$ F. Faupel, P.W. Hüppe and K. Rätzke, Phys. Rev. Lett. 65, 1219 (1990).

${ }^{46}$ H.R. Schober, C. Oligschleger and B.B. Laird, J. Noncryst. Sol. 156-158, 965 (1993).

${ }^{47}$ C. Oligschleger and H.R. Schober, Solid State Commun. 93, 1031 (1995).

${ }^{48}$ H. Miyagawa, Y. Hiwatari, B. Bernu and J.P. Hansen, J. Chem. Phys. 88, 3879 (1988).

49 G. Wahnström, Phys. Rev. A 44, 3752 (1991).

${ }^{50}$ J.M. Delaye and Y. Limoge, J. Phys. 1 France 3, 2079 (1993).

51 A. Heuer and R.J. Silbey, Phys. Rev. Lett. 70, 3911 (1993).

52 S. N. Taraskin and S. R. Elliott, Phys. Rev. B 56, 8605 (1997).

${ }^{53}$ R.W.G. Wyckoff, Crystal Structures (Krieger, Malabar, FL, 1982), p. 36 .

${ }^{54}$ C.A. Angell, J. Chem. Phys. Solids 49, 863 (1988).

${ }^{55}$ S.W. de Leeuw, J.W. Perram and E.R. Smith, Proc. Roy. Soc. A 373, 27, 1980, S.W. de Leeuw, J.W. Perram and E.R. Smith, Proc. Roy. Soc. A 373, 56, 1980.

${ }^{56}$ Harwell Subroutine Library, AERE Harwell, Didcot, UK.

${ }^{57}$ ESSL-Subroutine Library package.

${ }^{58}$ R. Fletcher and C.M. Reeves, Comput. J. 7, 149 (1964).

${ }^{59}$ D.L. Griscom, J. Non-Cryst. Solids 40, 211 (1980).

${ }^{60}$ D. Heinemann, and W. Mader, in Proc. 11th European Congress on Microscopy (EUREM 96), Dublin, 1996.

61 J.M. Carpenter and D.L. Price, Phys. Rev. Lett. 54, 441 (1985), D.L. Prince and J.M. Carpenter, J. Non-Cryst. Solids 92, 153 (1987).

${ }^{62}$ U. Buchenau, M. Prager, N. Nücker, A.J. Dianoux, N. Ah- 
mad, and W.A. Phillips, Phys. Rev. B 34, 5665 (1986).

${ }^{63}$ H.R. Schober and C. Oligschleger, Phys. Rev. B 53, 11469 (1996).

${ }^{64} \mathrm{C}$. Oligschleger, unpublished.
$\mathrm{K}$

$$
\begin{array}{r}
\mathrm{T}=3500 \mathrm{~K} \\
\mathrm{~T}=0 \mathrm{~K}
\end{array}
$$$$
\mathrm{T}=870
$$

\begin{tabular}{c|c|c|c|c|c|c}
\hline \hline $\mathrm{CN}$ & $\mathrm{Si}$ & $\mathrm{O}$ & $\mathrm{Si}$ & $\mathrm{O}$ & $\mathrm{Si}$ & $\mathrm{O}$ \\
\hline 0 & - & 0.000 & - & - & - & - \\
\hline 1 & - & 0.045 & - & 0.025 & - & 0.022 \\
\hline 2 & 0.000 & 0.901 & - & 0.972 & - & 0.976 \\
\hline 3 & 0.094 & 0.047 & 0.045 & 0.003 & 0.039 & 0.002 \\
\hline 4 & 0.905 & 0.005 & 0.955 & - & 0.961 & - \\
\hline 5 & 0.001 & 0.000 & - & - & - & - \\
\hline \hline
\end{tabular}

TABLE I. Distributions of the coordination numbers $(C N)$ for both silicon and oxygen at $T=3500 \mathrm{~K}, 870 \mathrm{~K}$ and $0 \mathrm{~K}$, above and well below the glass transition temperature, respectively. 
FIG. 1. Element specific self diffusion constants in $\mathrm{SiO}_{2}$ for $\mathrm{Si}(\diamond)$ and $\mathrm{O}(+)$ plotted versus temperature.

FIG. 2. Total pair correlation function $g(r)$ (a) and the partial pair correlation functions (b-d) in amorphous $\mathrm{SiO}_{2}$ at $T=270 \mathrm{~K}$.

FIG. 3. Comparison between the calculated pair distribution function $\mathrm{d}(\mathrm{r})$ and the experimenta- fesult by D. Heinemann using electron scattering (see Ref.60).

FIG. 4. Bond angle distribution in silica glasses. The $\mathrm{O}-\mathrm{Si}-\mathrm{O}$ distribution has the peak-position at $109^{\circ}$ and a FWHM of $10^{\circ}$. The $\mathrm{Si}-\mathrm{O}-\mathrm{Si}$ distribution has the peak-position at $145^{\circ}$ and a FWHM of $25^{\circ}$.

FIG. 5. Density of states $\mathrm{Z}(\nu)$ vs. $\nu$ obtained by calculation of the Fourier transform of the displacement autocorrelation function using the equation of motion (EOM) method (solid line) and diagonalization (dashed line). The dotted line displays the Debye DOS.

FIG. 6. Specific heat $\mathrm{c}_{\mathrm{V}}$ as $\mathrm{c}_{\mathrm{V}} / \mathrm{T}^{3}$ in units of $\left[\mu \mathrm{J} / g K^{4}\right]$ vs. $T$ in K. Plot is double-logarithmic. Solid line is the specific heat with Debye-correction and dotted line without correction. Diamonds are experimental values of specific heat $c_{p}$ by Zeller and Pohl.

FIG. 7. Participation ratio of the vibrational eigenmodes for glasses with $N=1944(\diamond)$ and $N=576(+)$ atoms.

FIG. 8. Averaged element specific contributions to the vibrational spectrum for 14 glasses with $N=1944$ atoms. Total DOS (solid line), O-contribution (dashed line) and Si-contribution (broken line) are plotted versus frequency in $\mathrm{THz}$.

FIG. 9. Weighted averaged element specific distribution of the angles $\alpha_{e b o n d s}$ between the atomic eigenvector and the bonds for all low-frequency modes. The full line show the angle-distribution found for silicon, the broken line for oxygen.

FIG. 10. The vibrational DOS (solid line) and the partial contributions for the projections of the relative atomic displacement onto the symmetry coordinates of the SiOSi-subunits with the contributions from rotations (dashed-dotted line), symmetric stretching (broken line), asymmetric stretching (dashed line) and from symmetric bending (dotted line) plotted versus frequency in $\mathrm{THz}$.
FIG. 11. The DOS (solid line) and the partial contributions for the projections of the relative atomic displacements onto the symmetry coordinates of the $\mathrm{SiO}_{4}$-subunits with the contributions from rotations (dashed-dotted line), symmetric stretching (broken line), asymmetric stretching (dashed line) and from bending in E-symmetry (dotted line) and in $\mathrm{F}_{2}$-symmetry (dashed-dot-dotted line) plotted versus frequency in $\mathrm{THz}$.

FIG. 12. Displacement of O vs. displacement of Si in single jumps. The line shows the calculated $\mathrm{O}$ displacement if one scales $\Delta R^{S i}$ by the square root of the mass ratio $\mathrm{O}$ to $\mathrm{Si}$ and by the ratio of the atomic numbers.

FIG. 13. Participation ratio for the quenching regimes vs. displacement. The line is a guide to the eye.

FIG. 14. Change of potential energies per atom averaged over an ensemble of $8 \mathrm{MD}$-runs at $T=870 \mathrm{~K}$ (a). Total displacements $\Delta \mathrm{R}^{2}$ over an ensemble of $8 \mathrm{MD}$-runs at $T=870 \mathrm{~K}$ (b).

FIG. 15. Distribution of the angle $\alpha_{\text {rbonds }}$ between the relaxations $r$ and the bonds of the atoms, full line Si-relaxation, dashed line O-relaxation. 


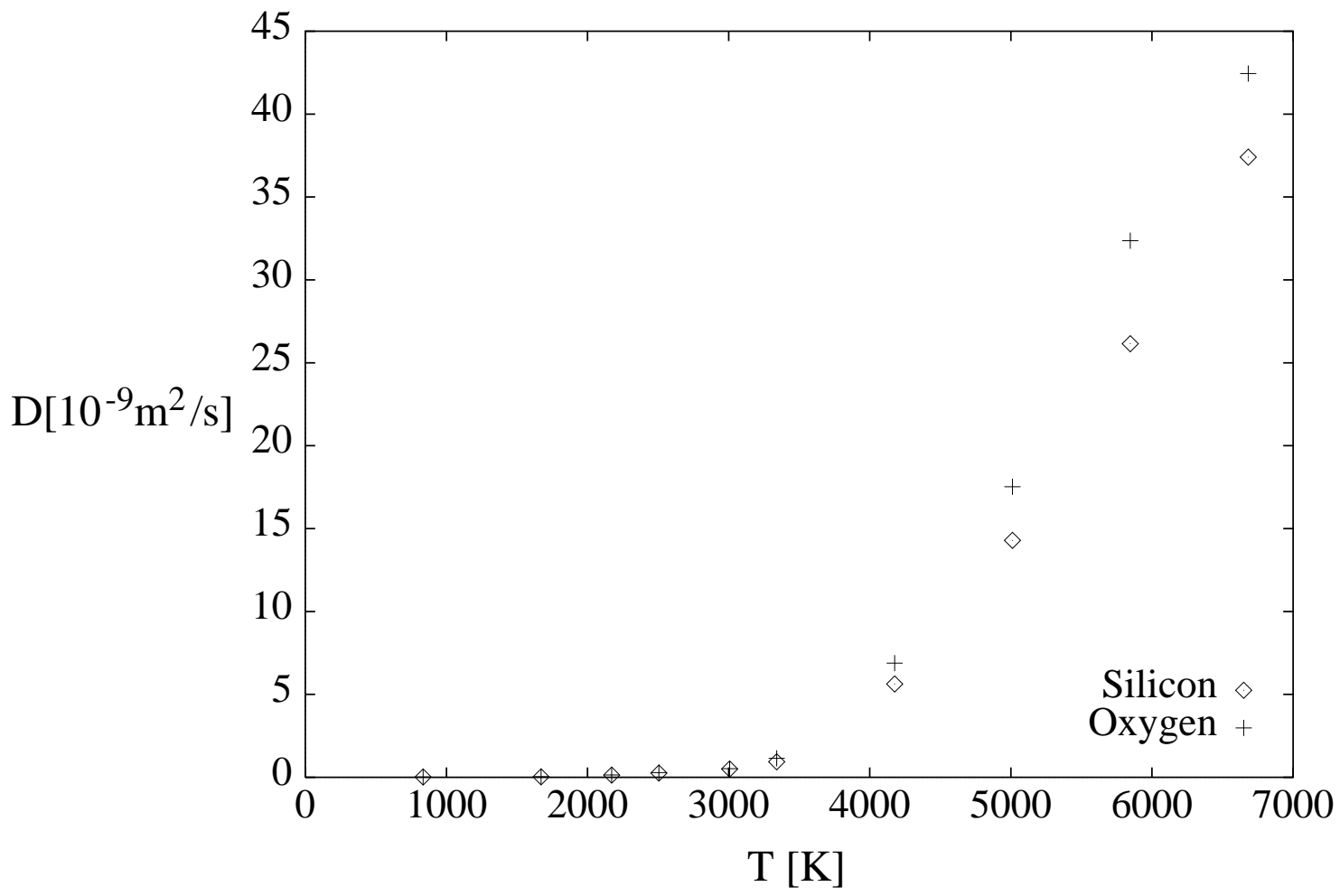




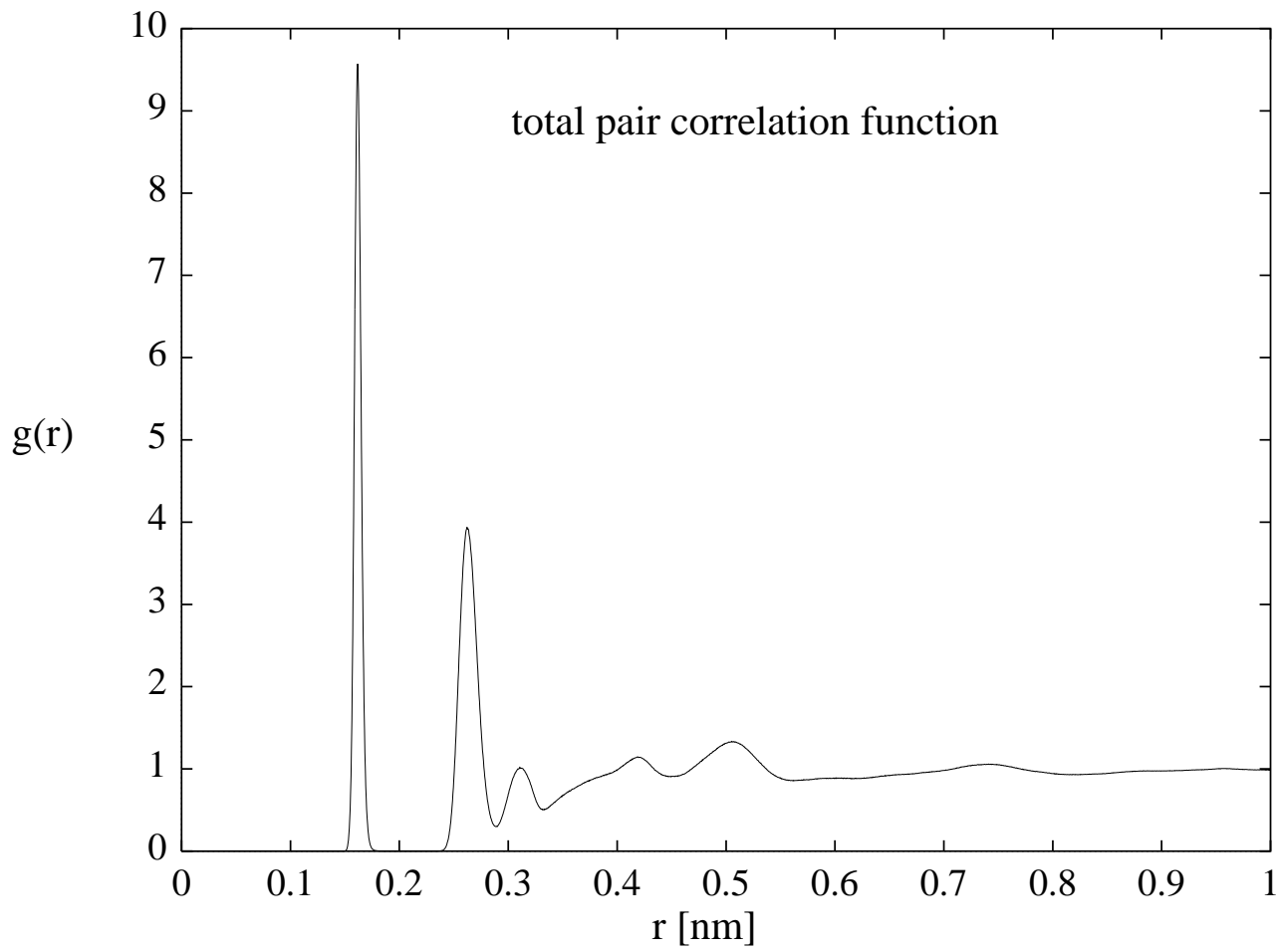




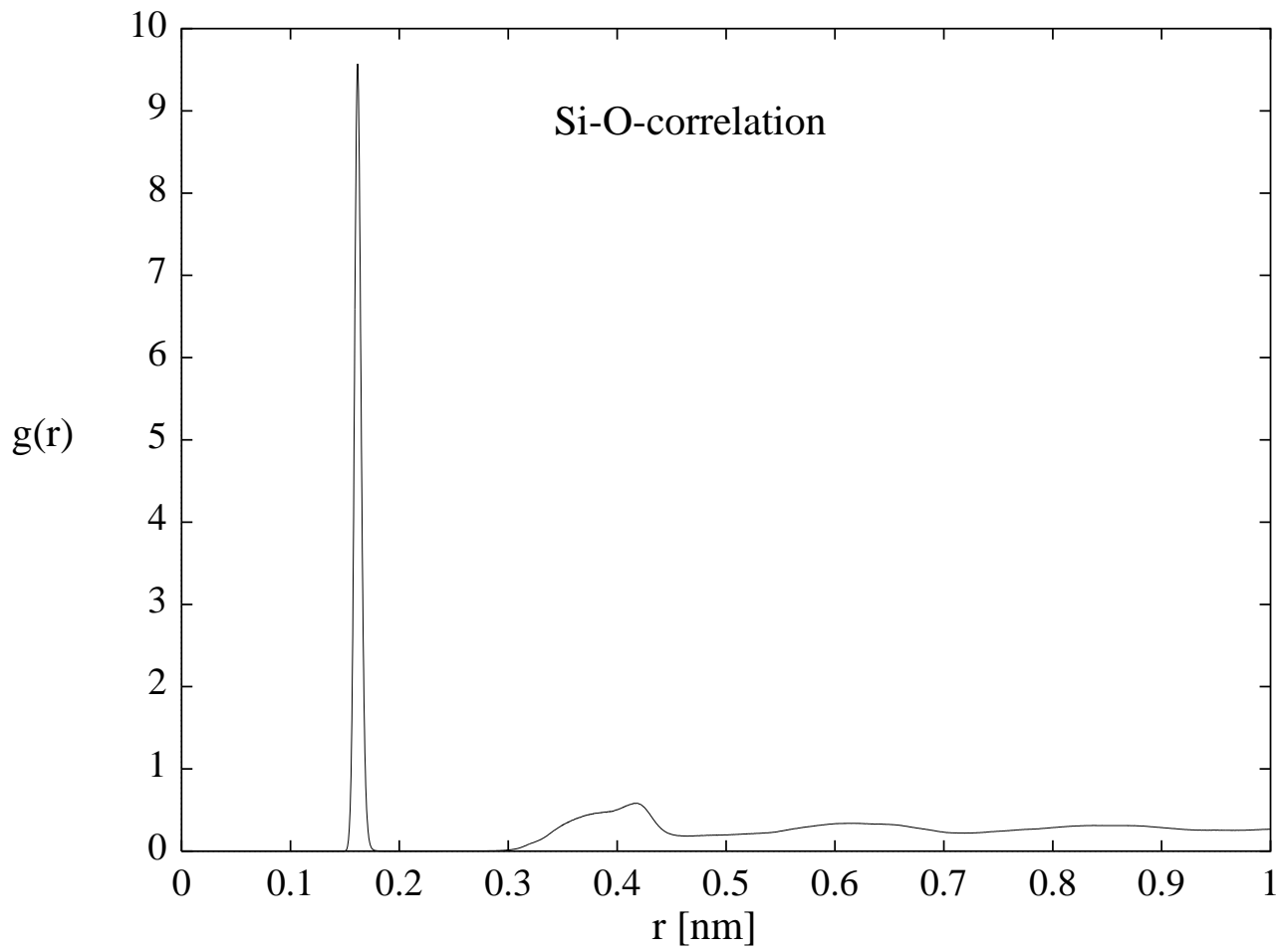




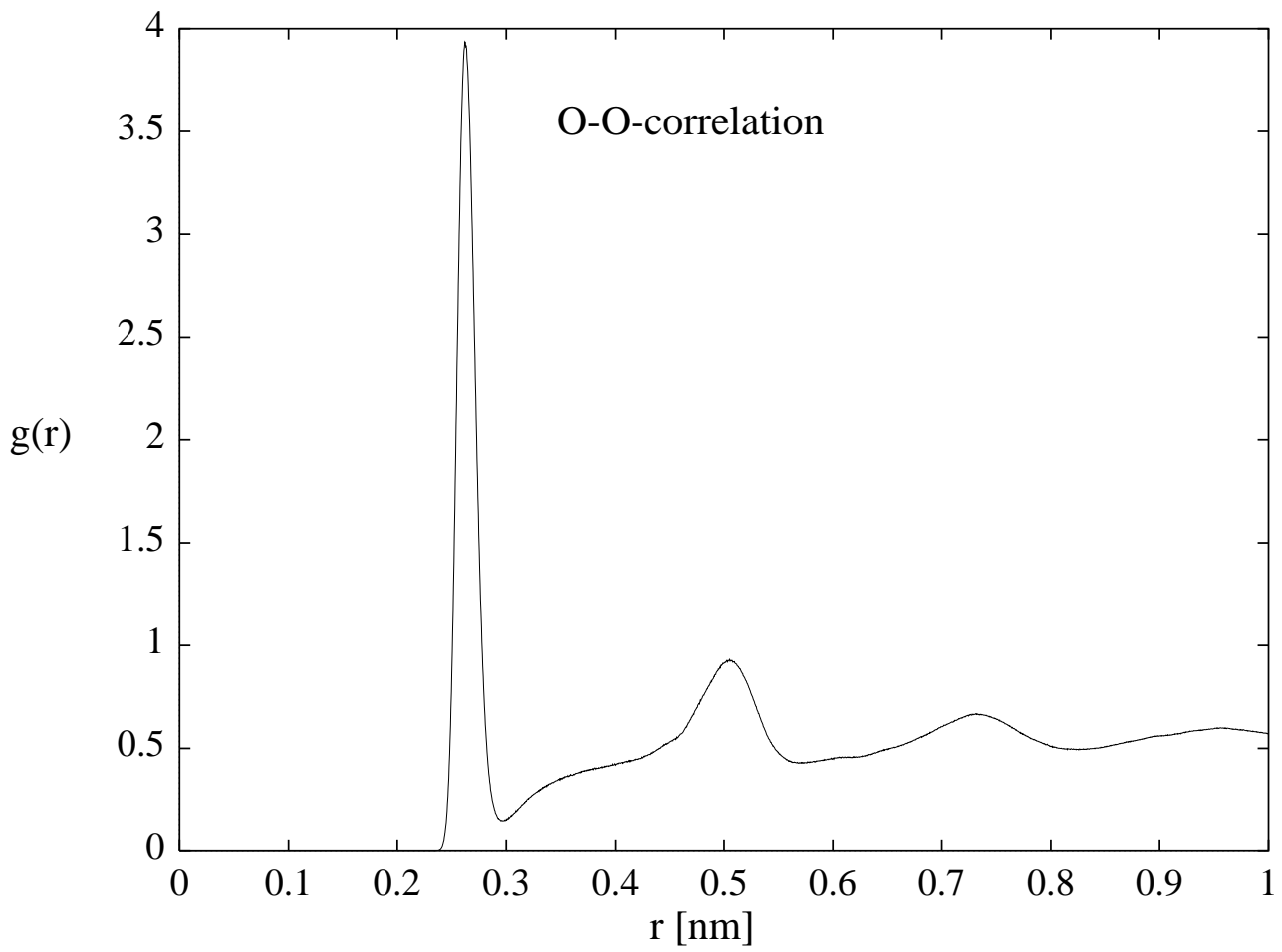




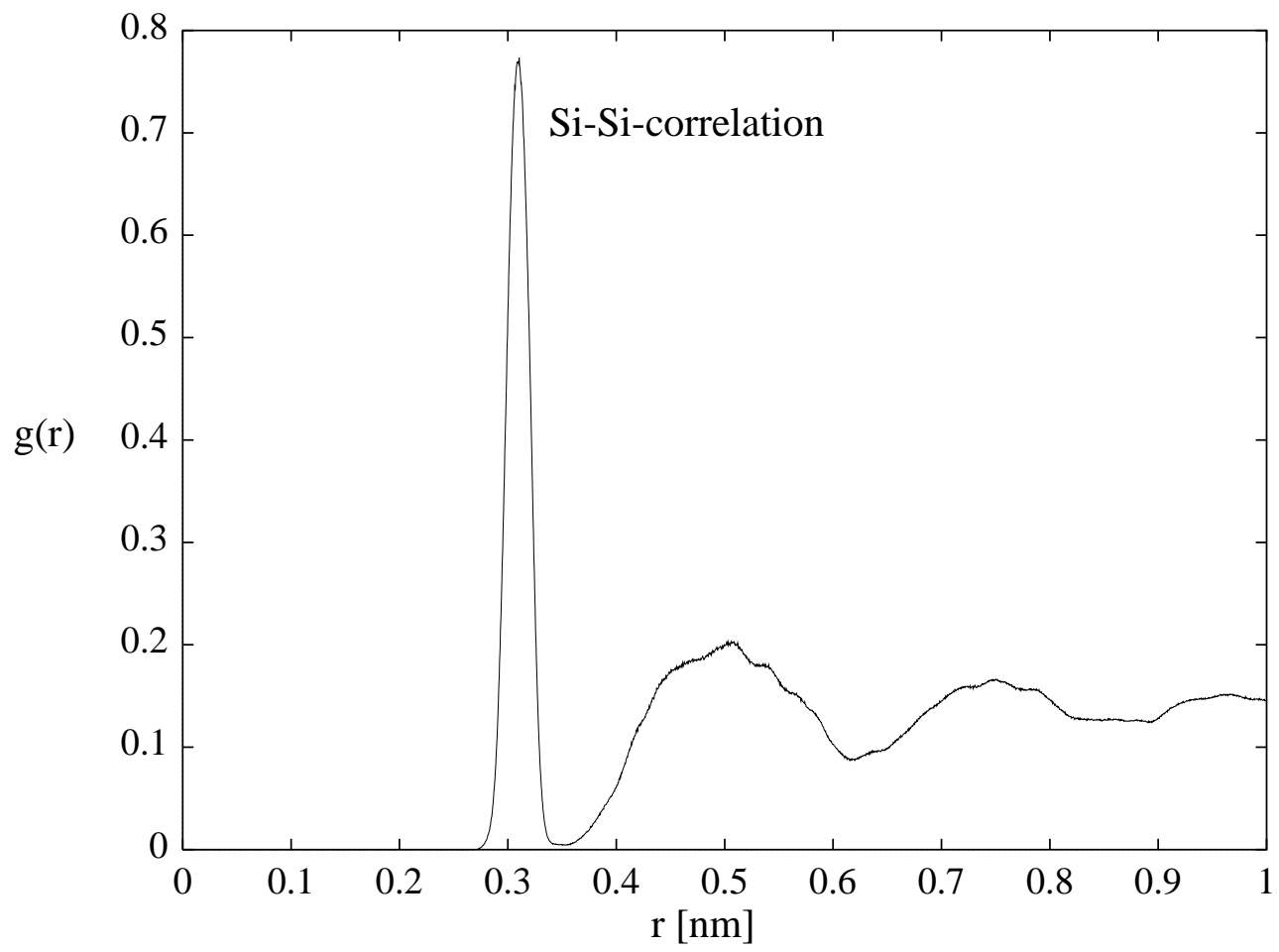




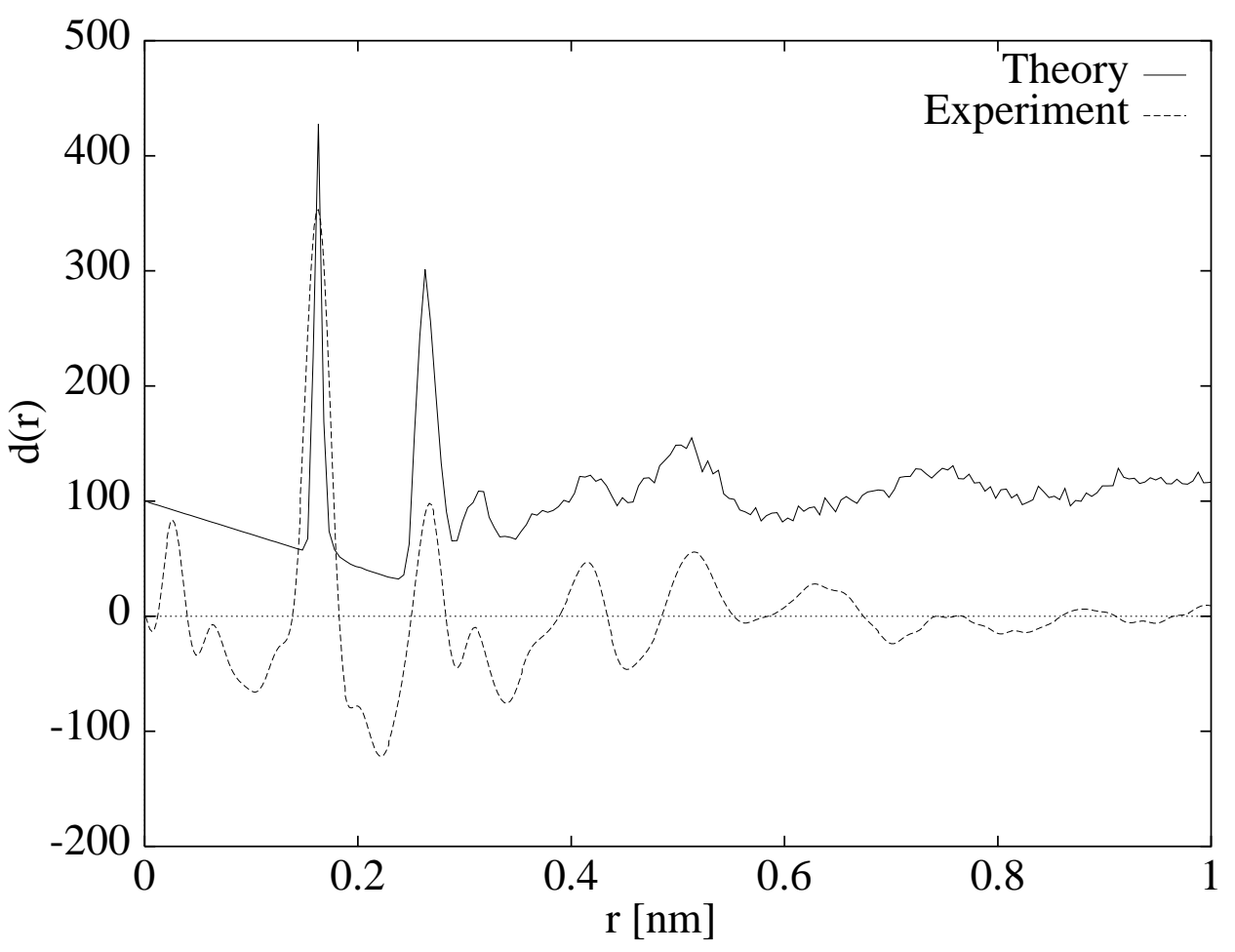




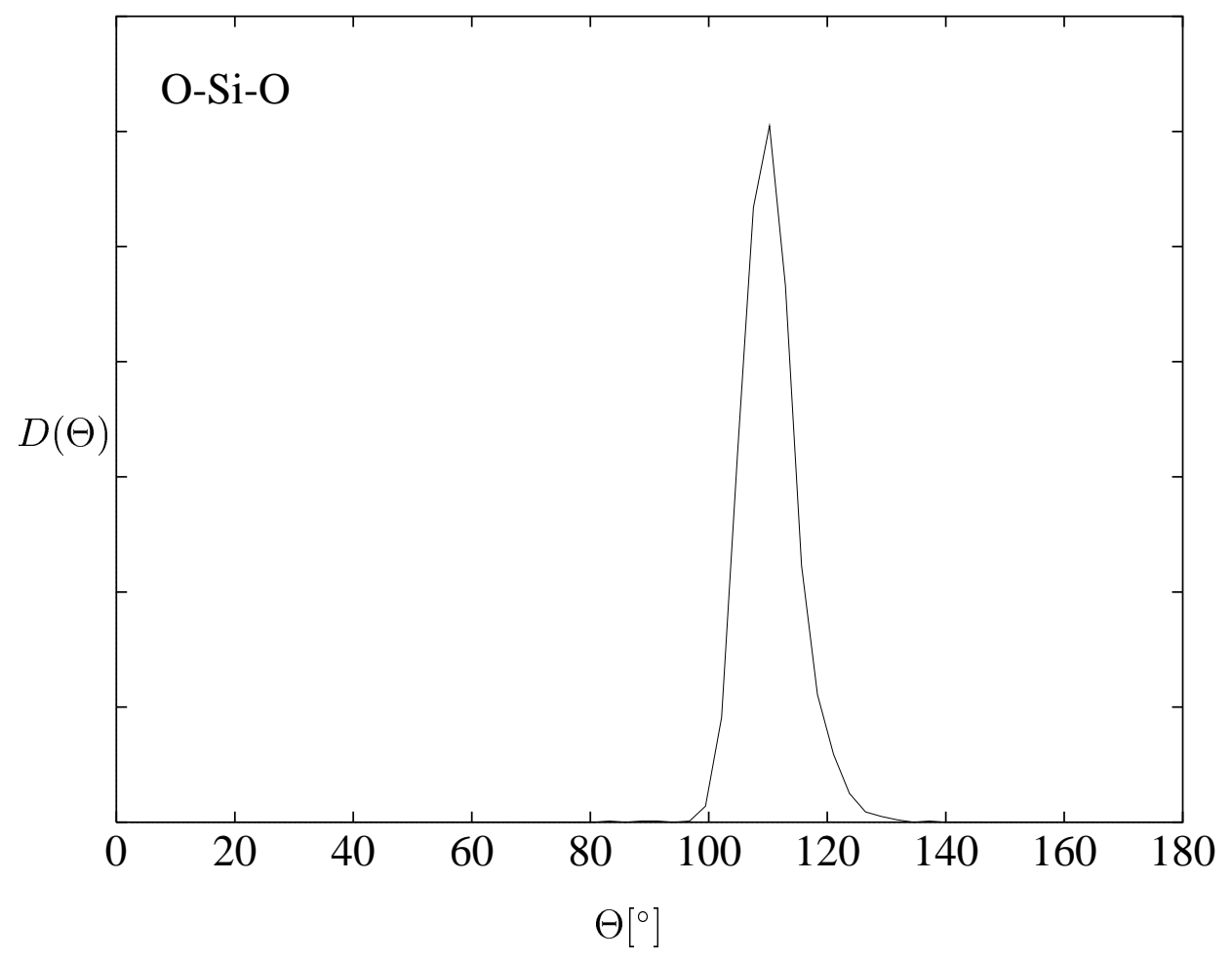




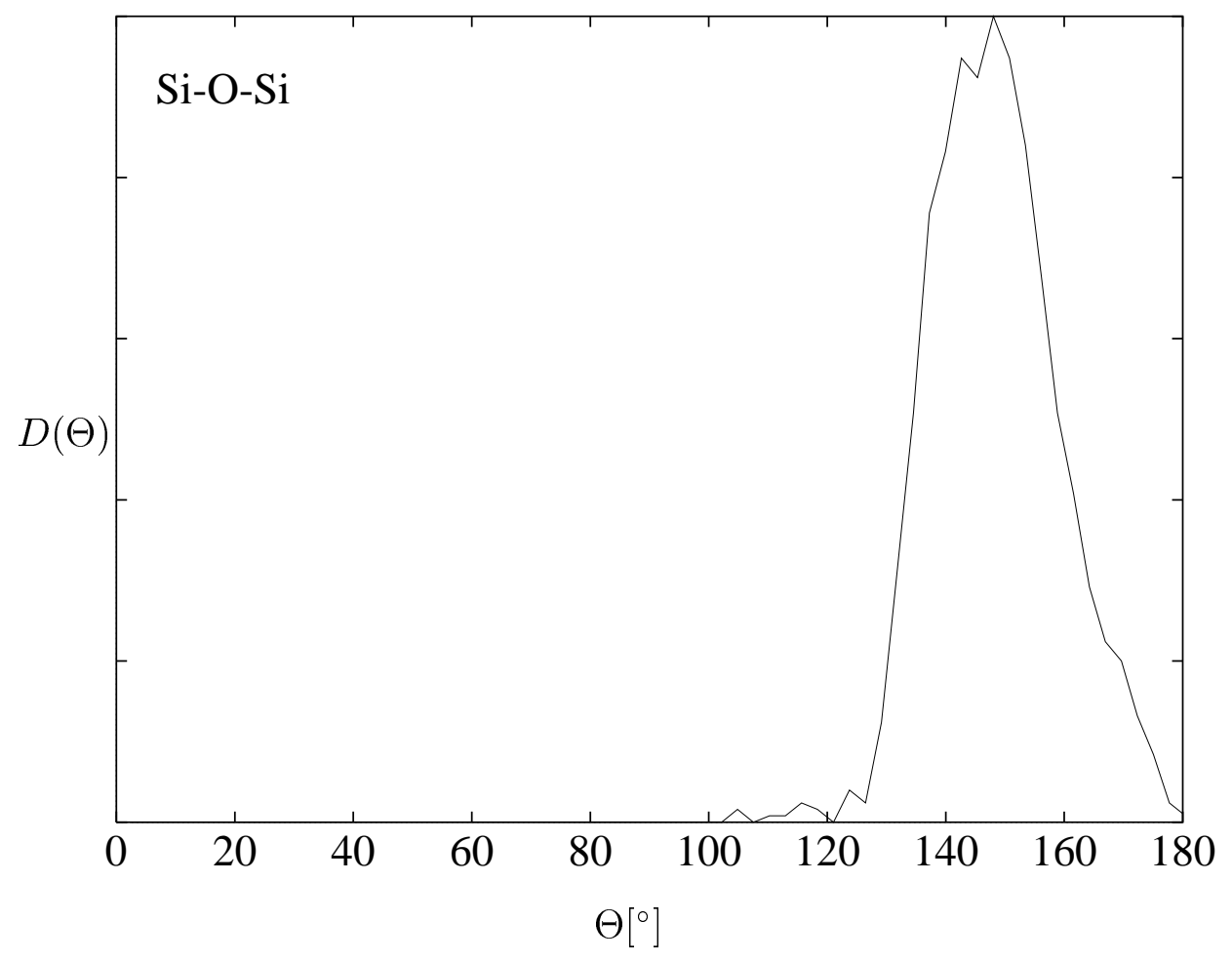




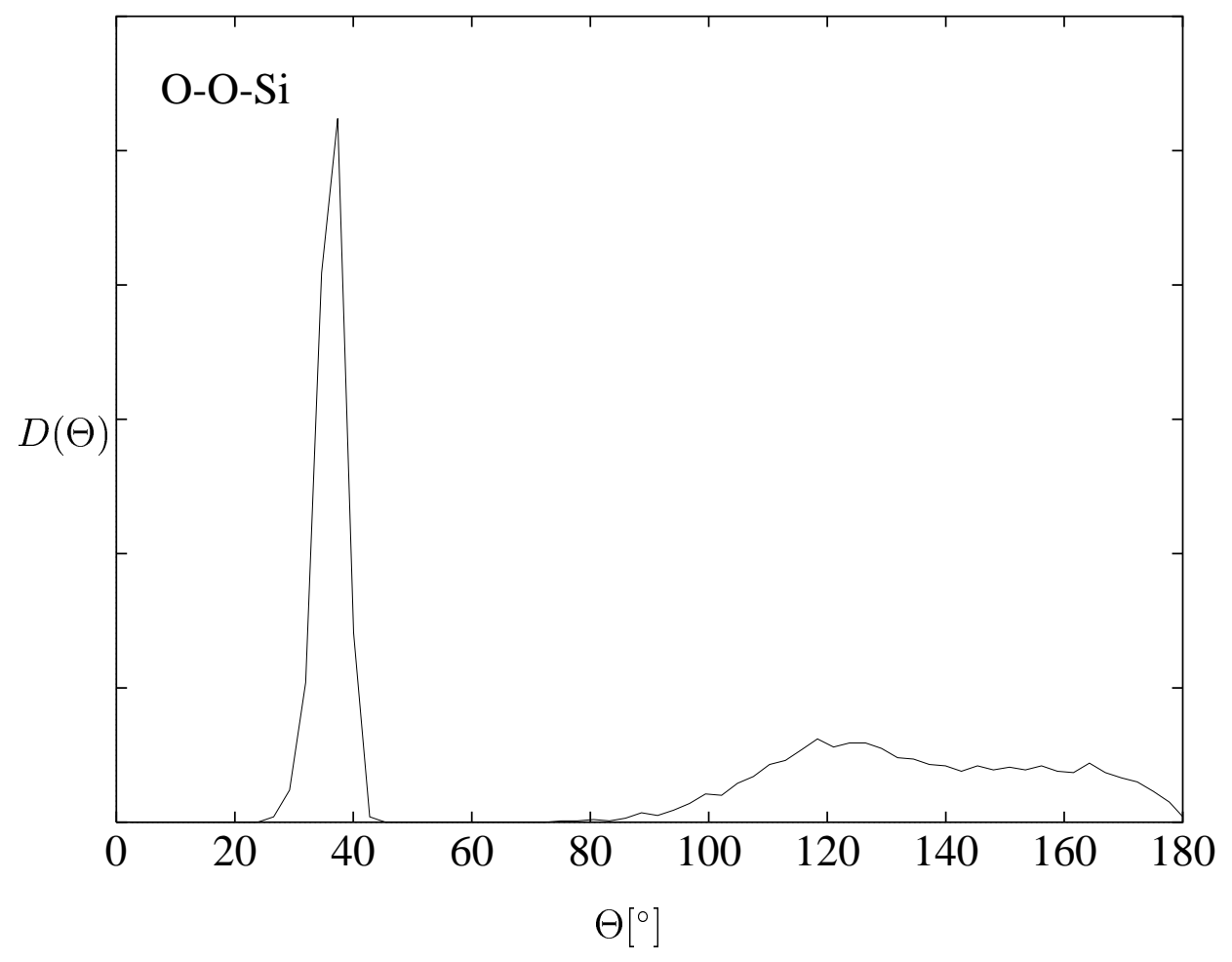




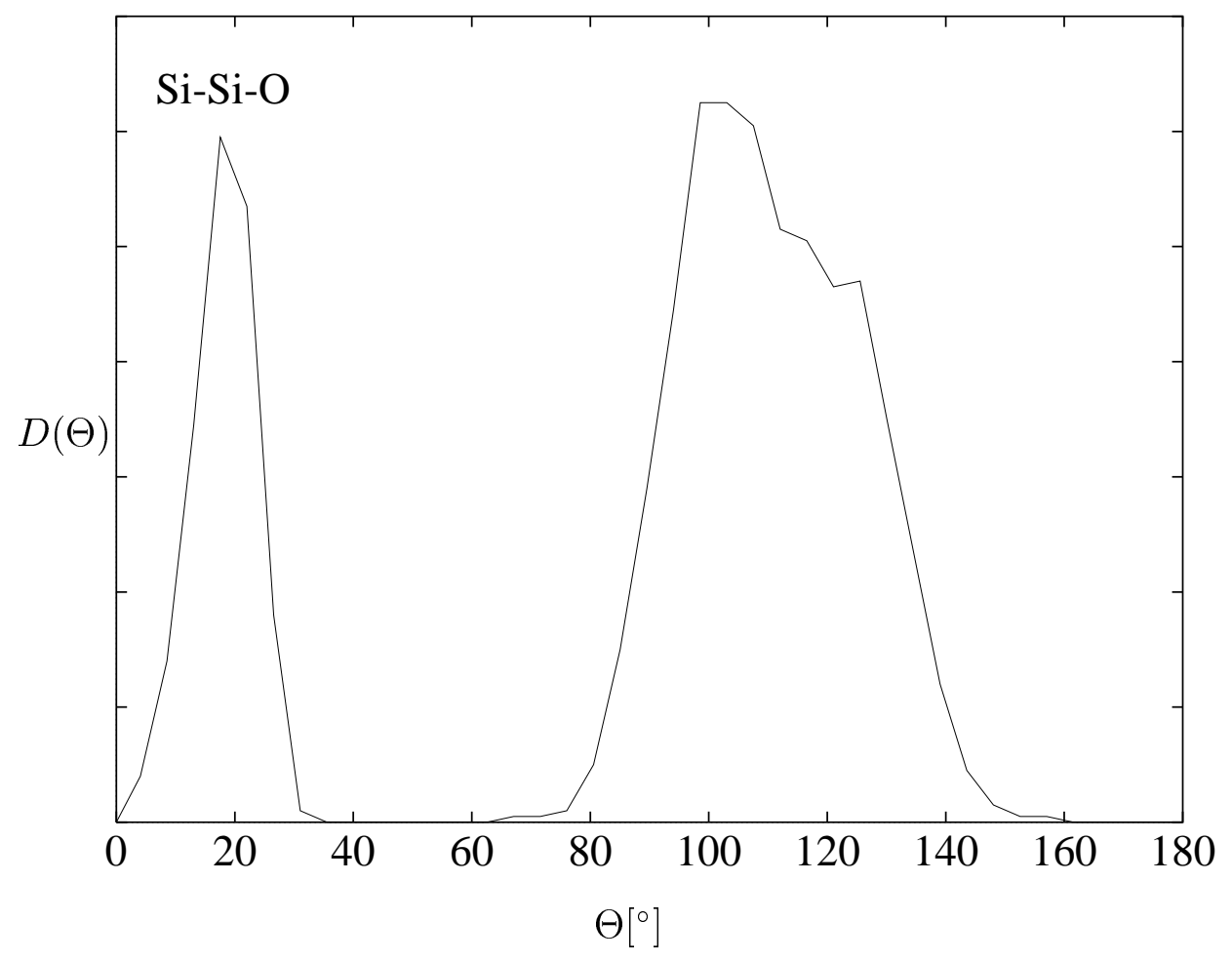




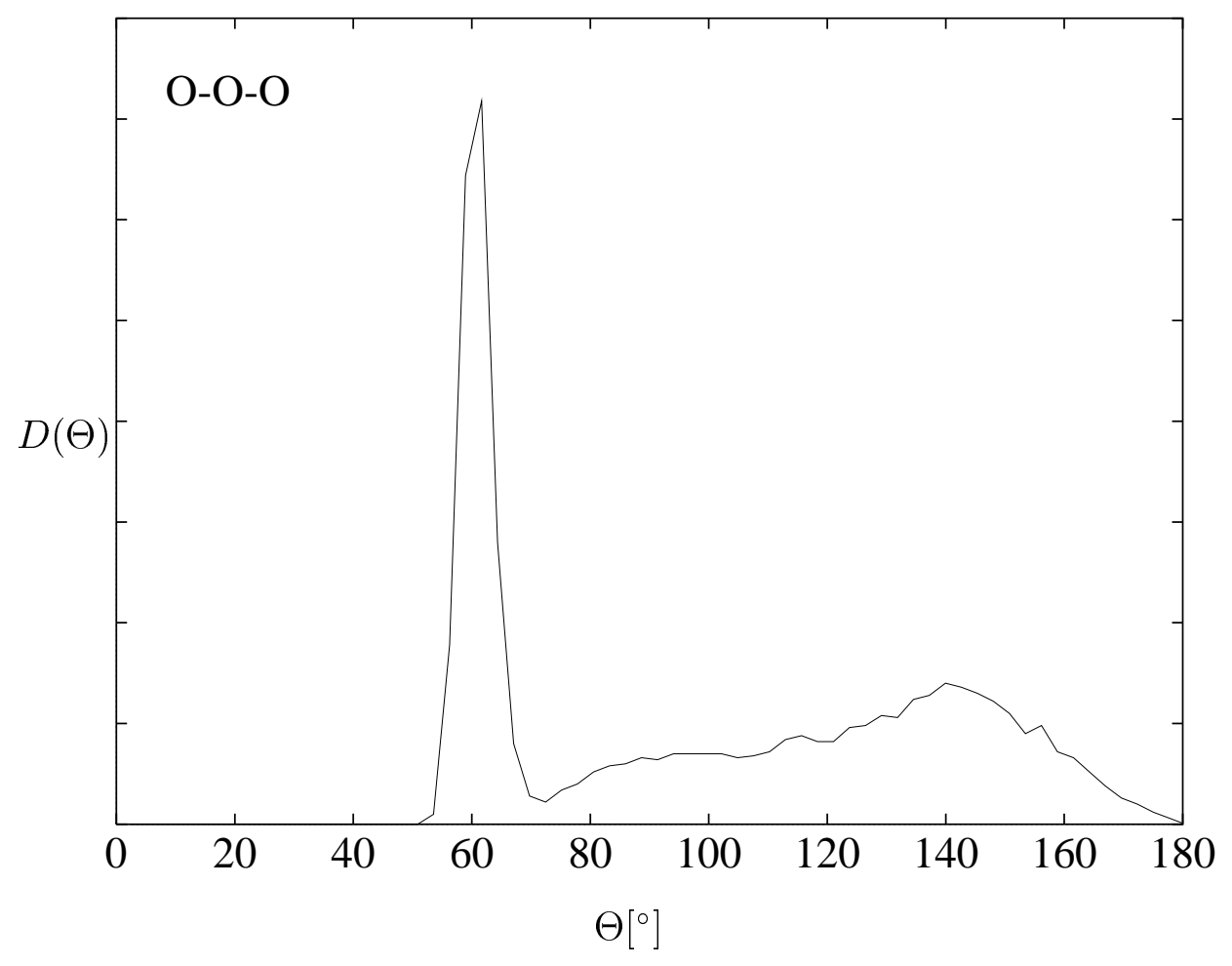




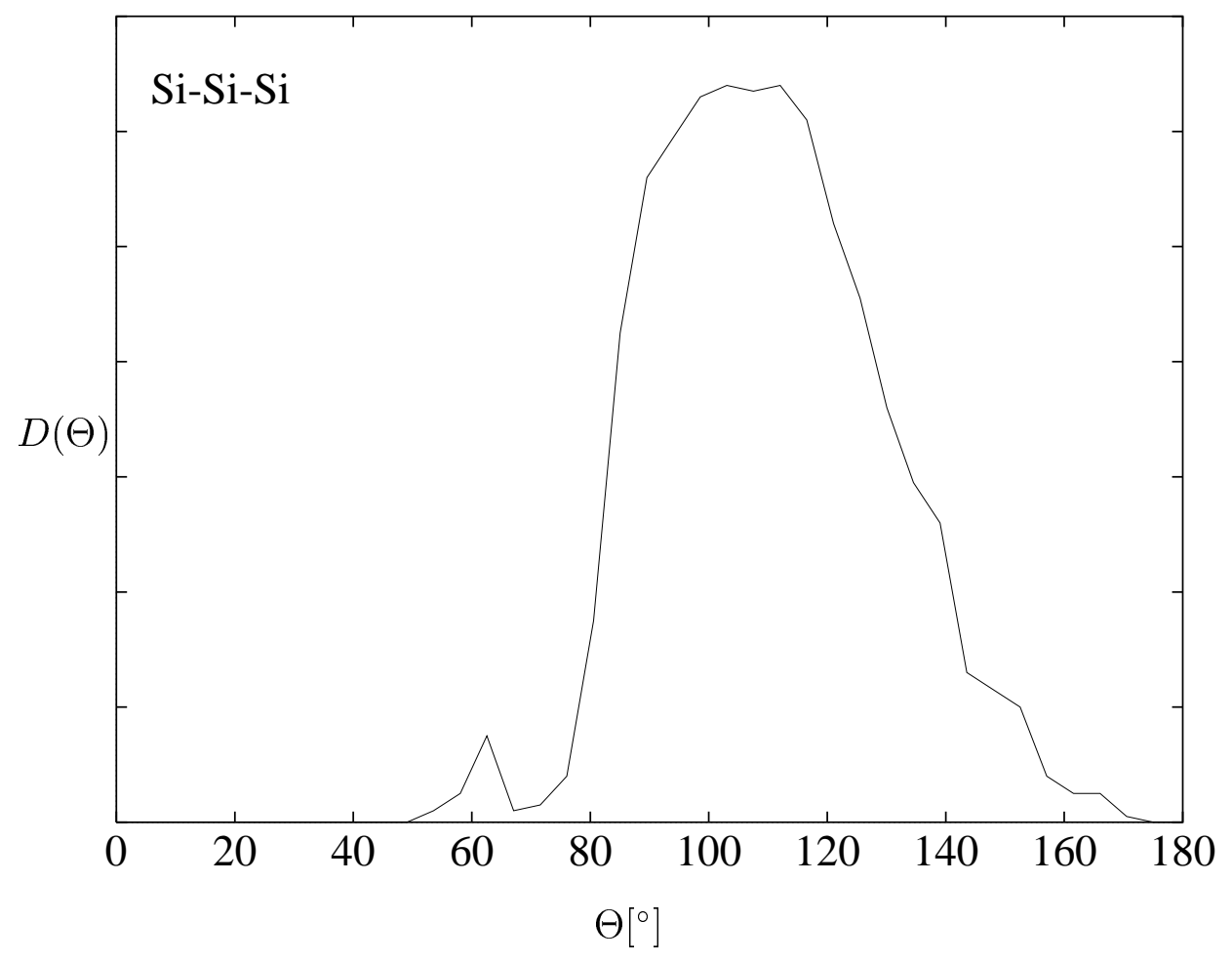




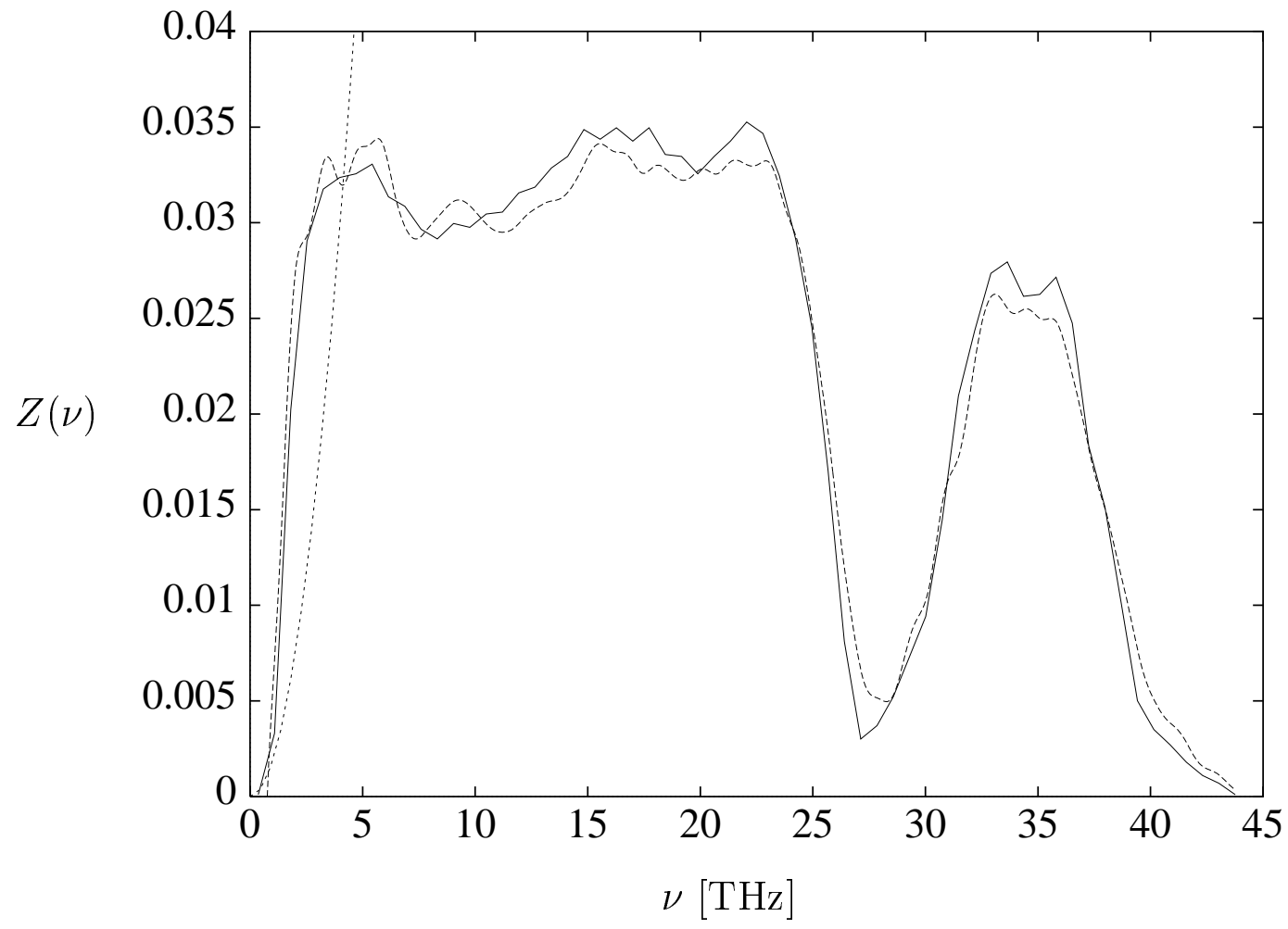




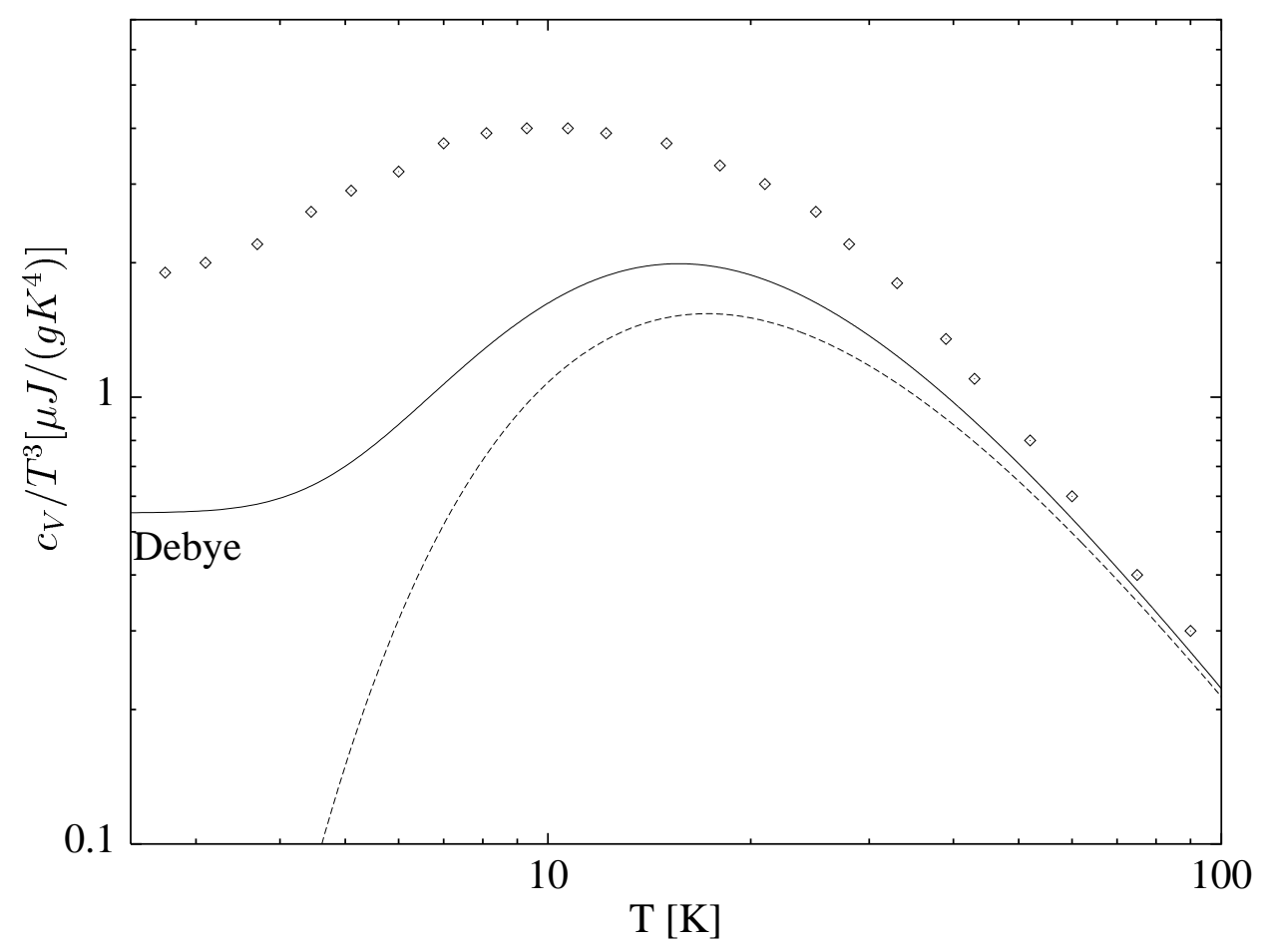




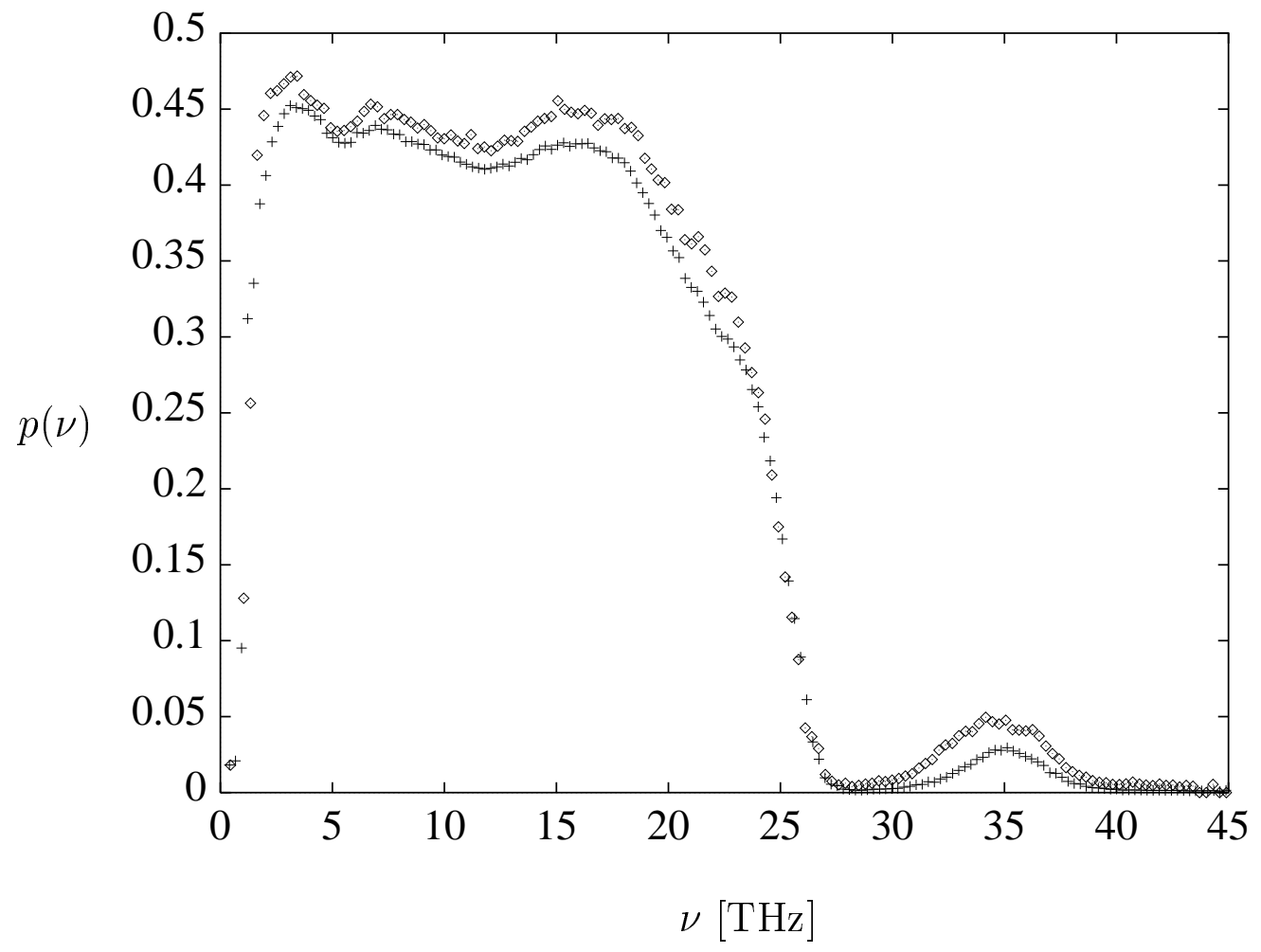




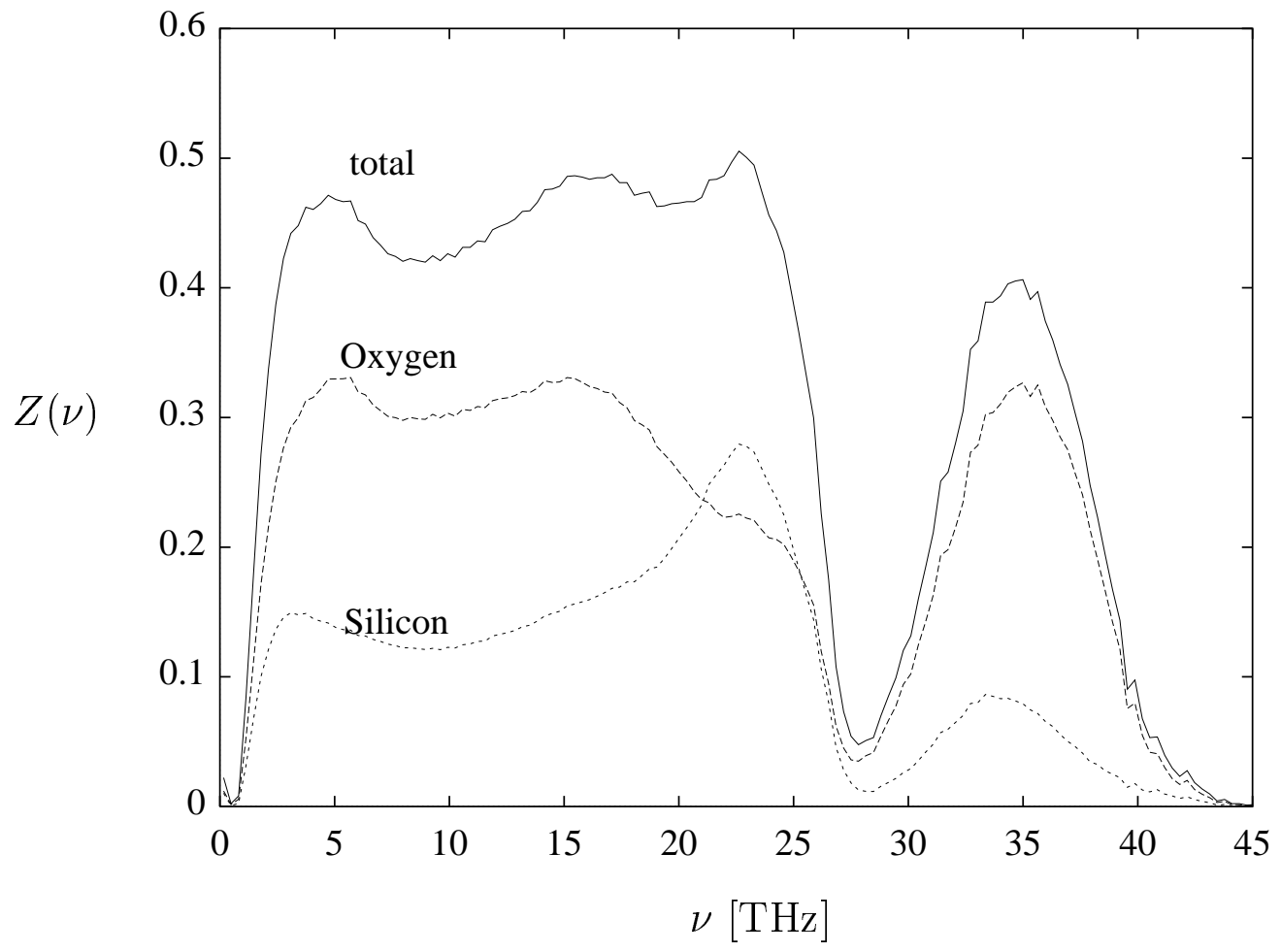




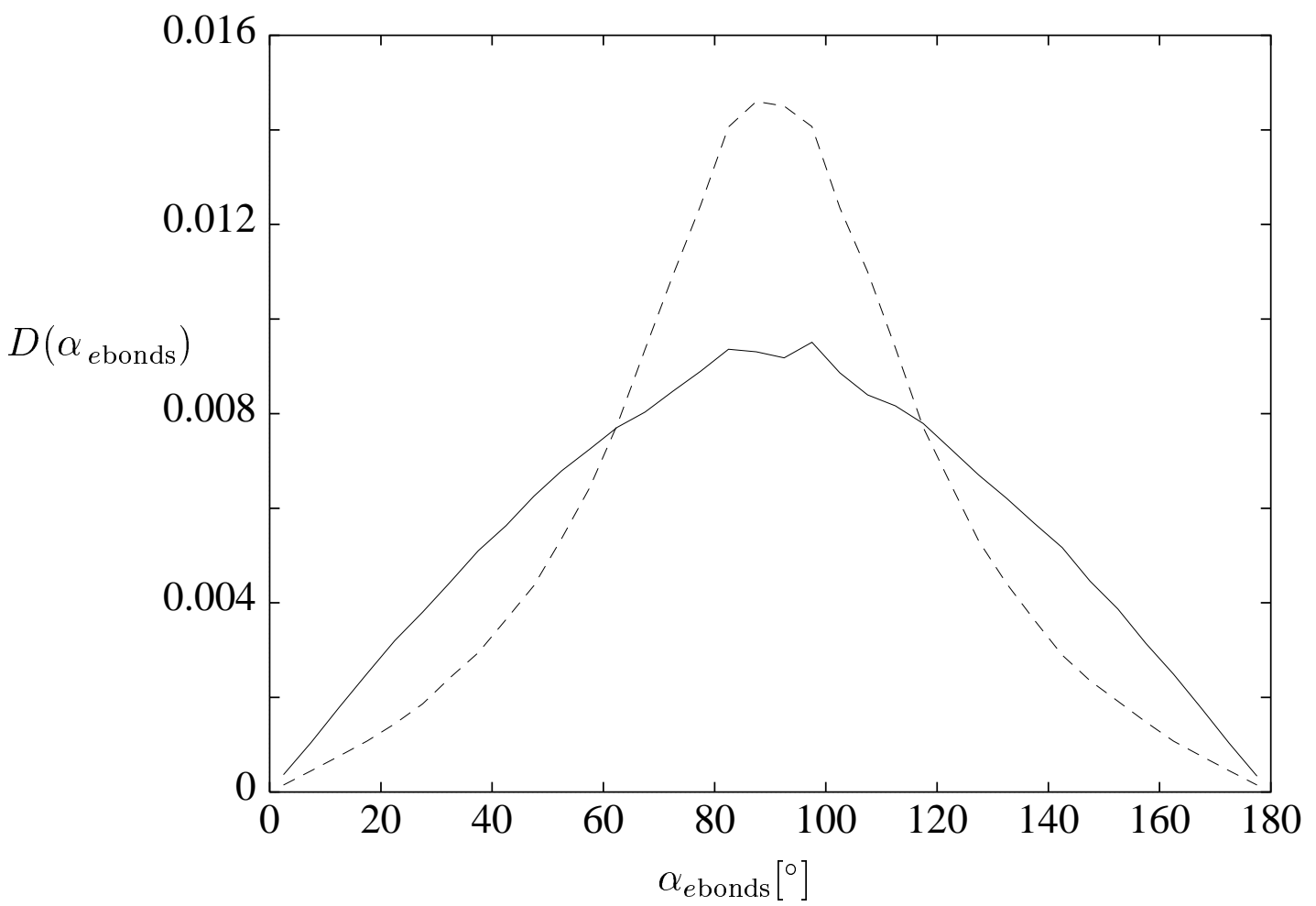




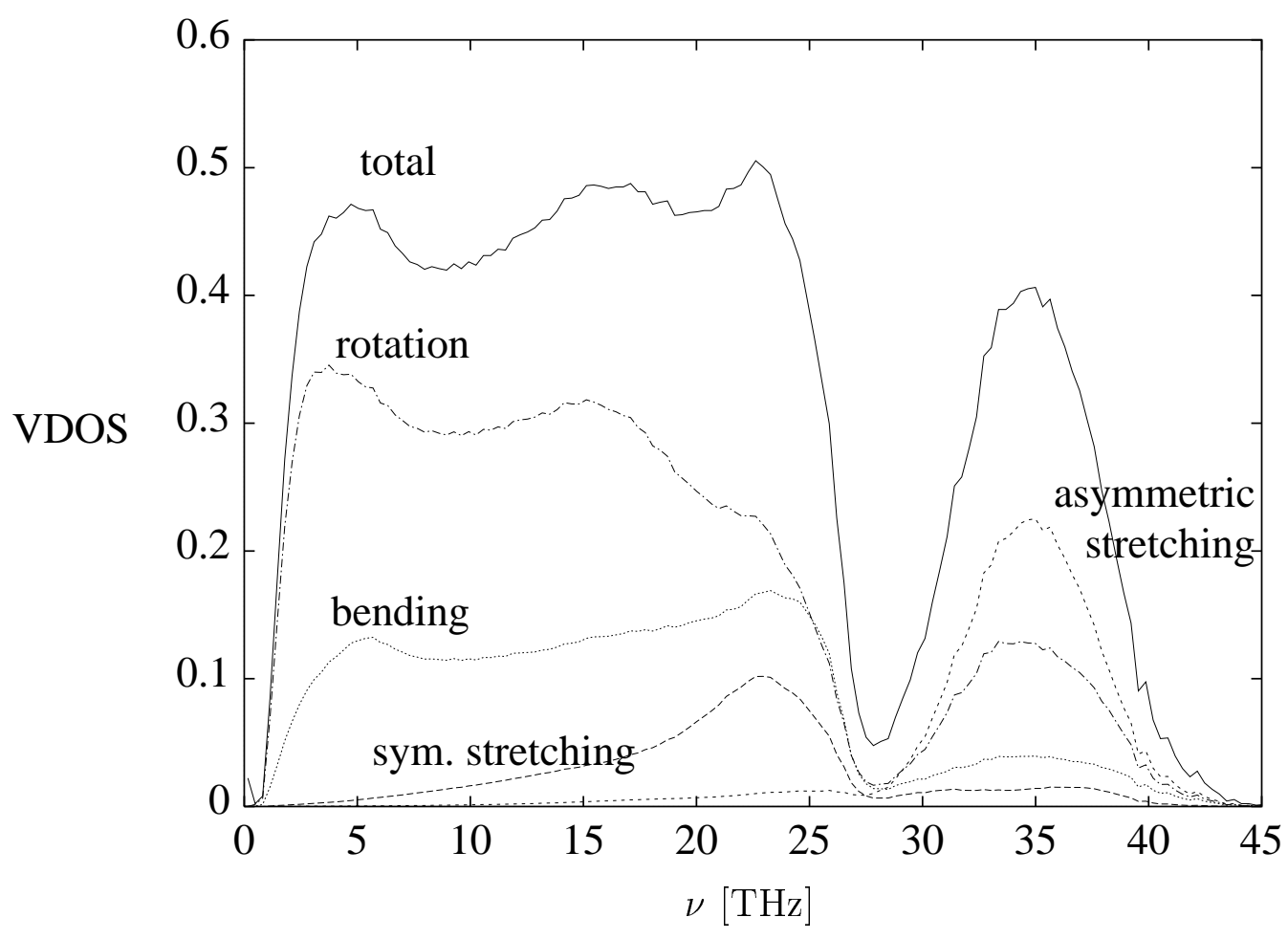




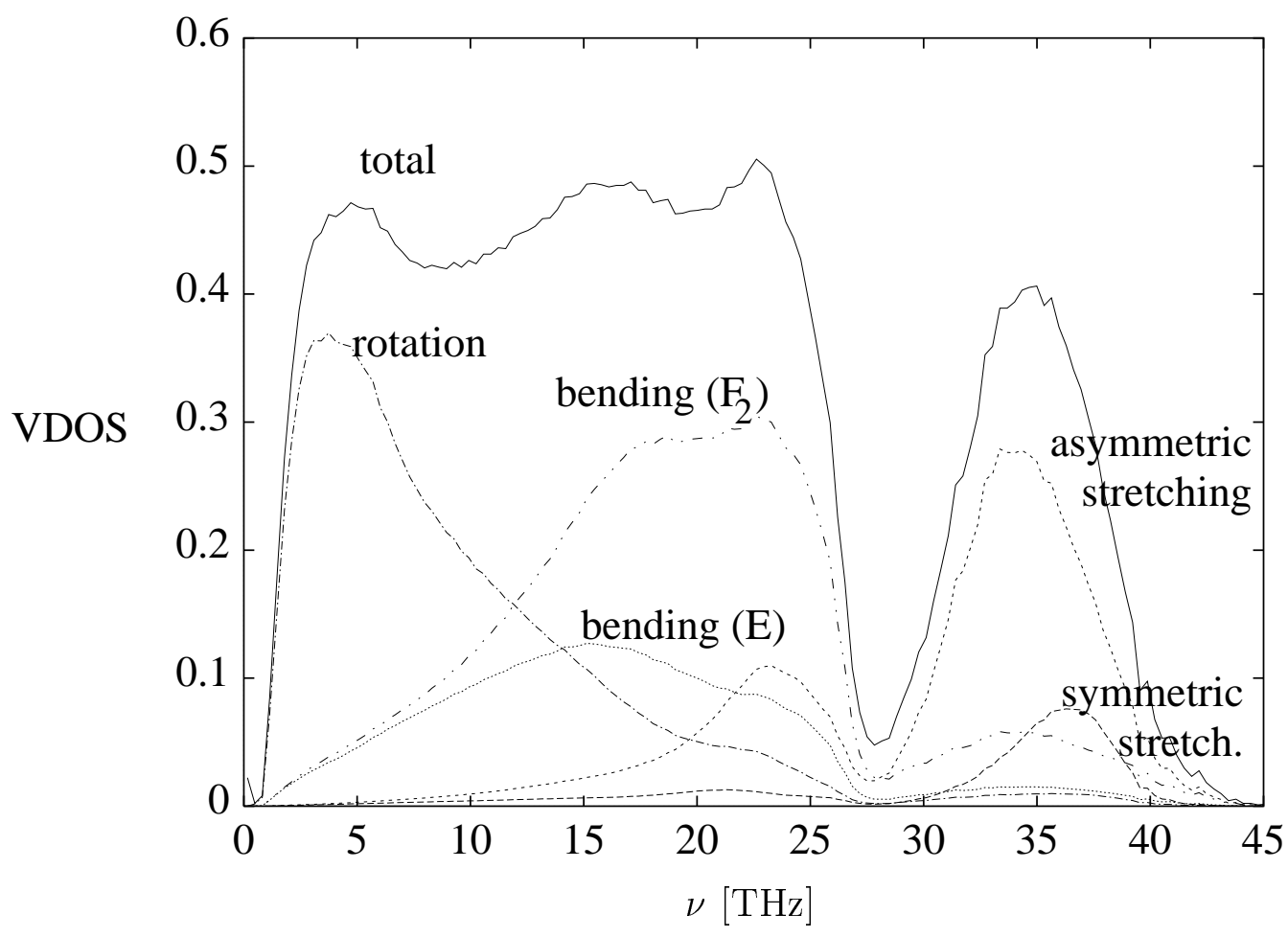




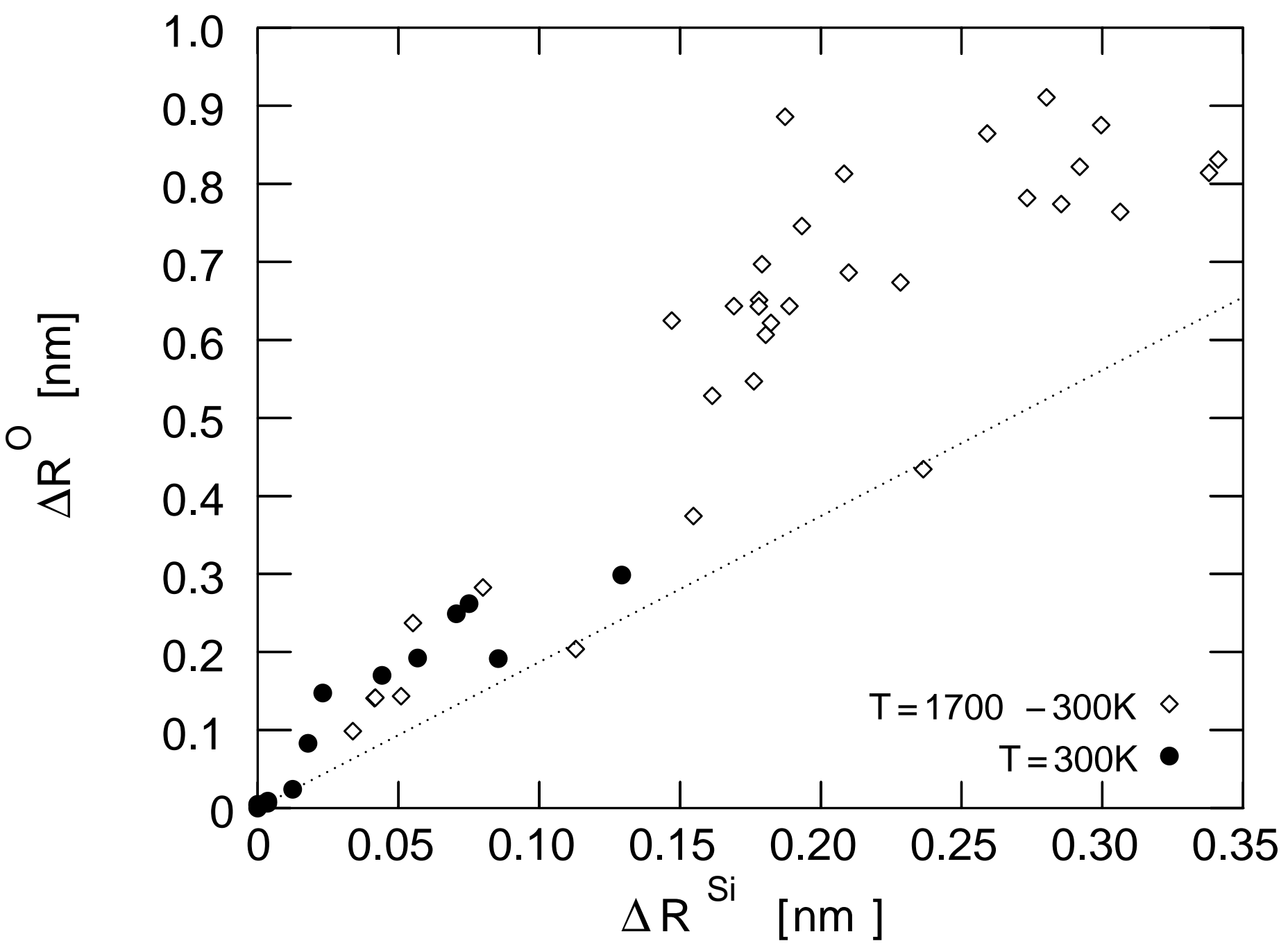




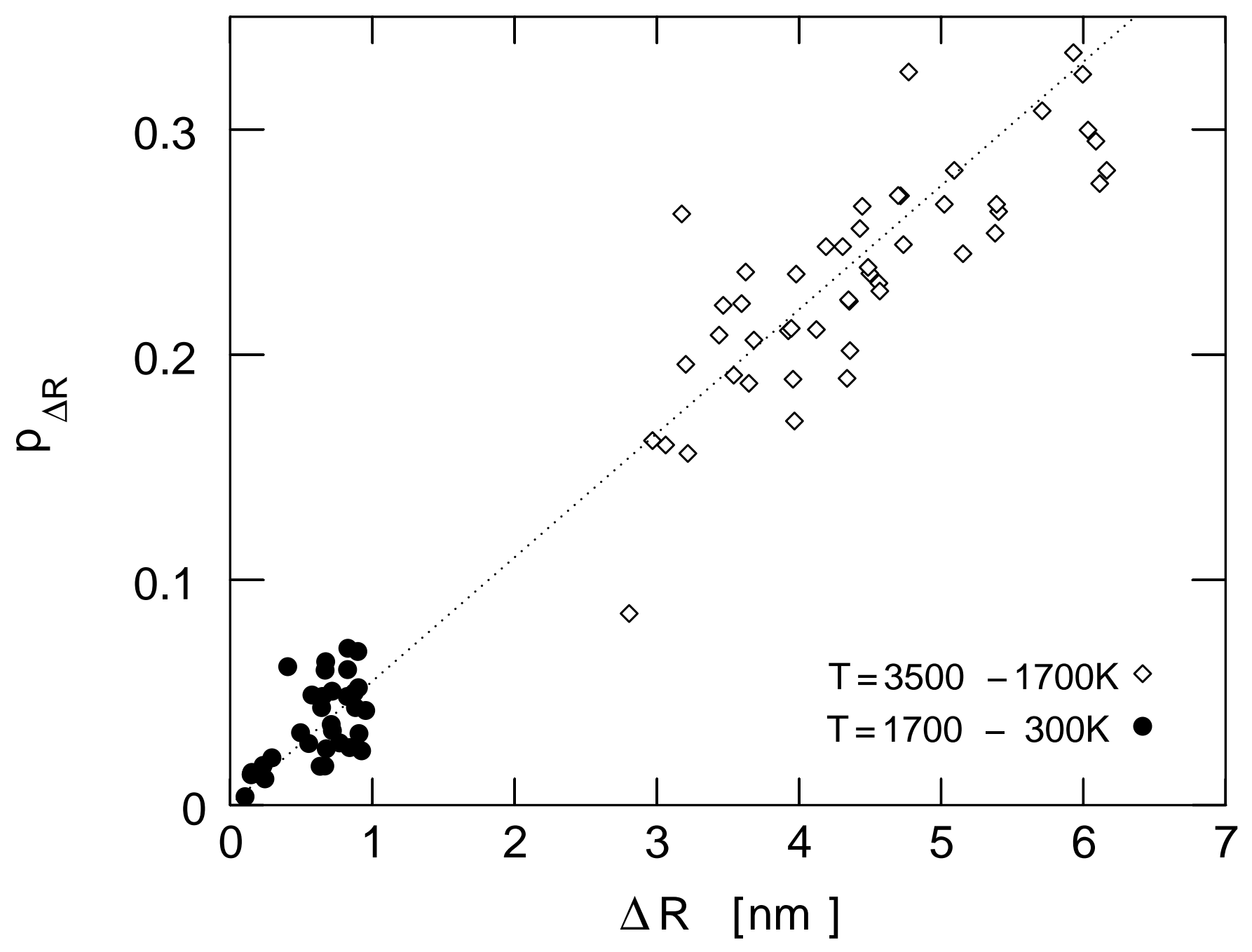




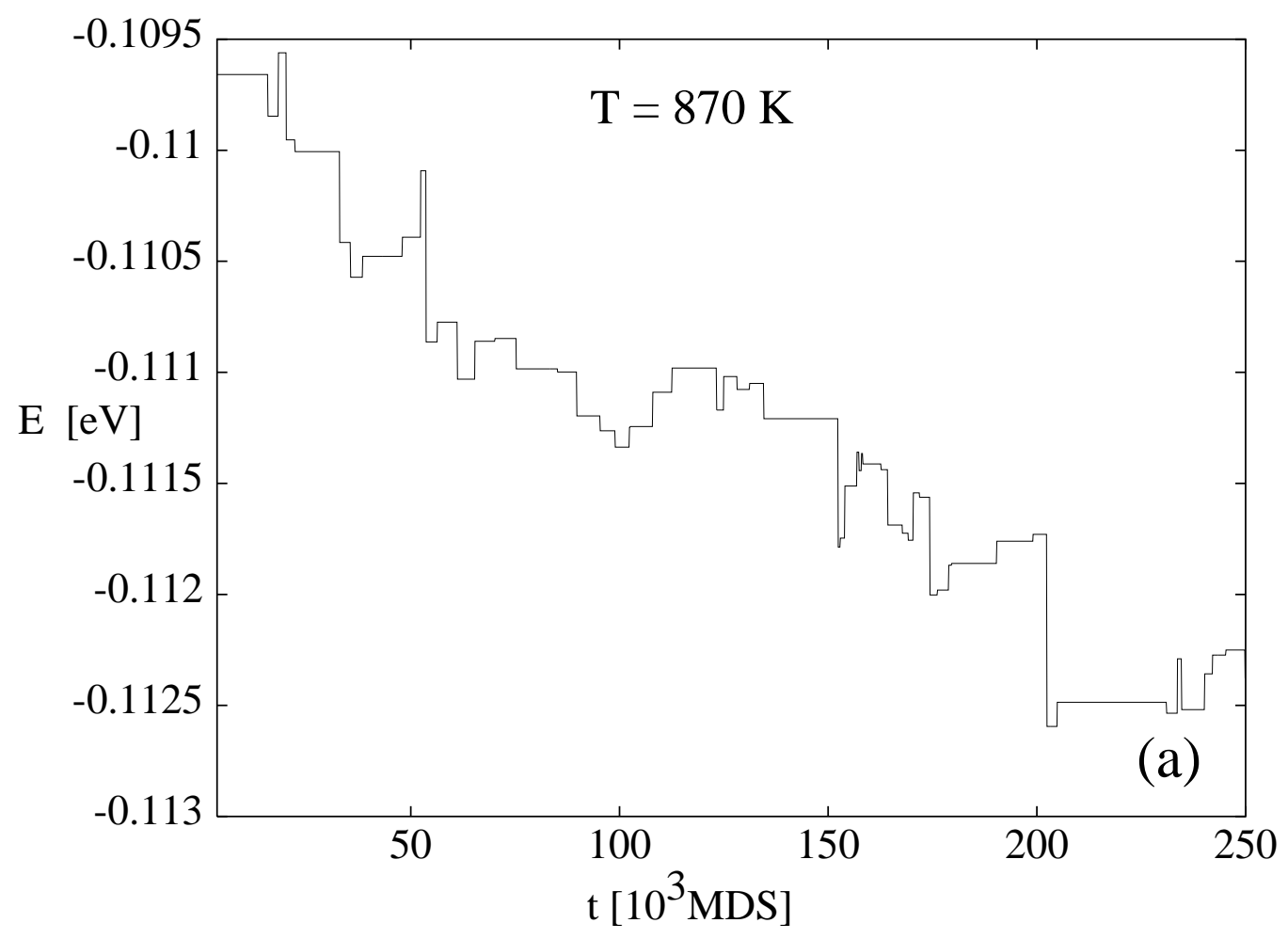




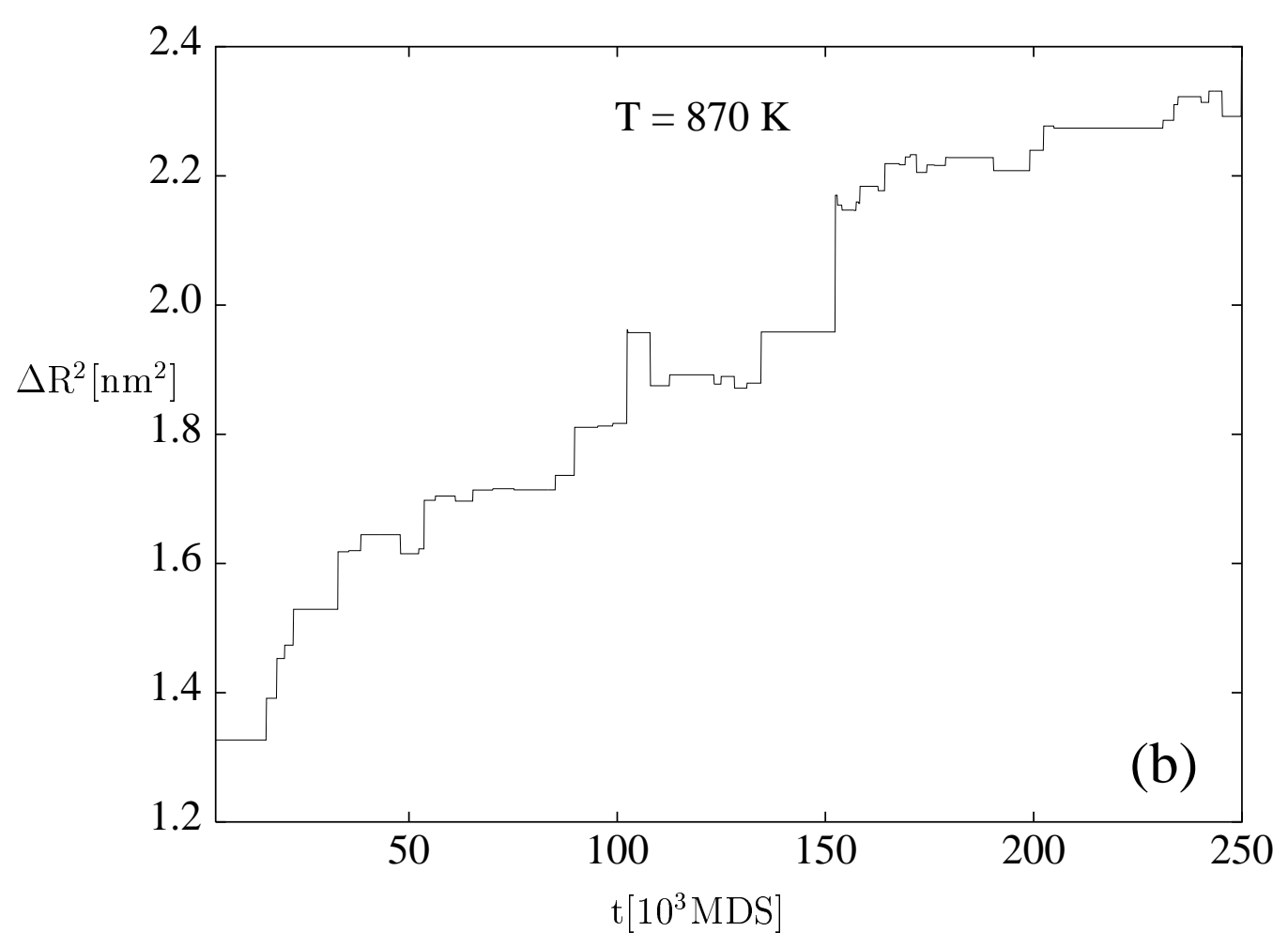




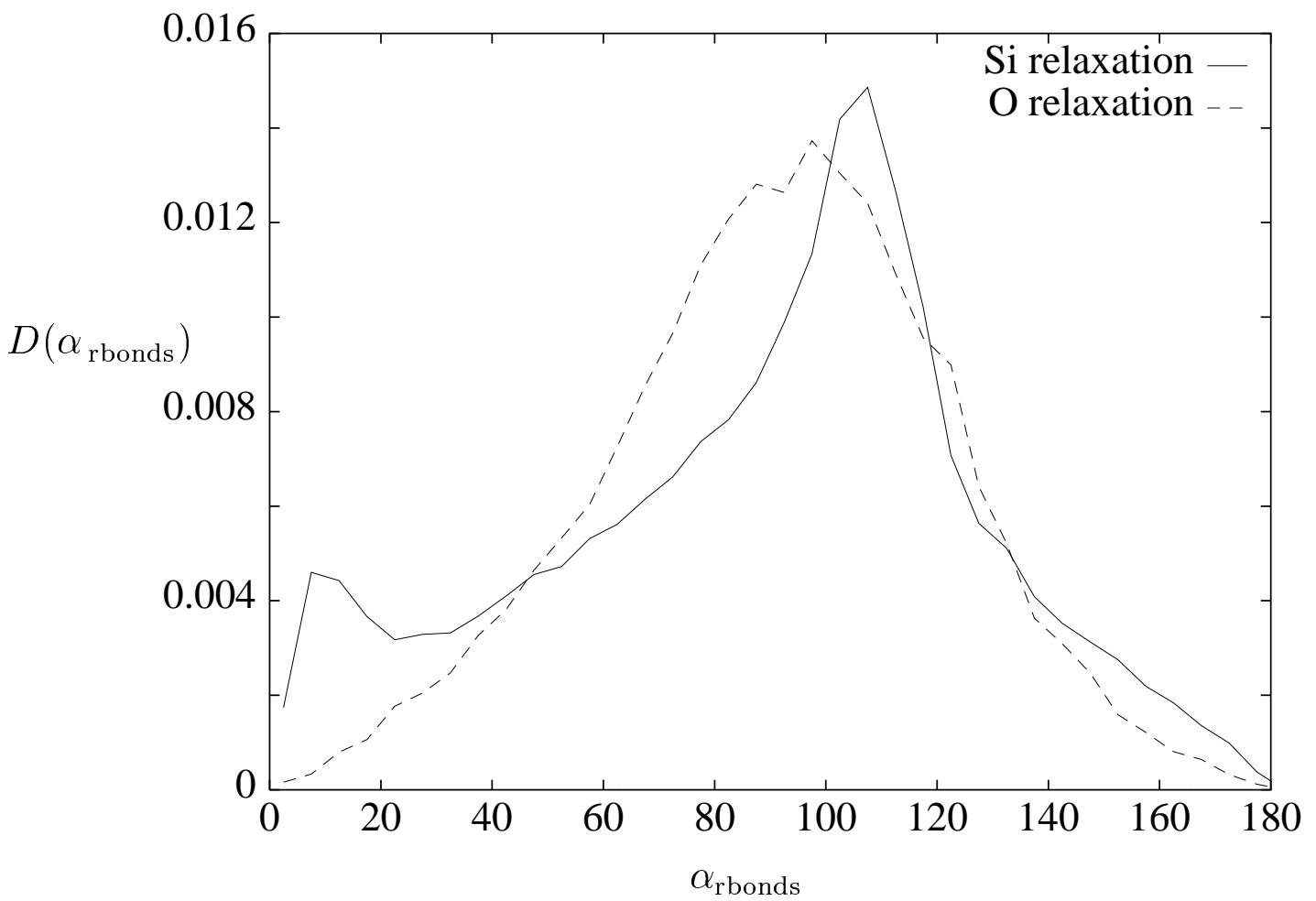

